\title{
PURELY 1-UNRECTIFIABLE METRIC SPACES AND LOCALLY FLAT LIPSCHITZ FUNCTIONS
}

\author{
RAMÓN J. ALIAGA, CHRIS GARTLAND, COLIN PETITJEAN, \\ AND ANTONÍN PROCHÁZKA
}

\begin{abstract}
For any compact metric space $M$, we prove that the locally flat Lipschitz functions separate points (of $M$ ) uniformly if and only if $M$ is purely 1-unrectifiable, resolving a problem posed by Weaver in 1999. We subsequently use this geometric characterization to answer several questions in Lipschitz analysis. Notably, it follows that the Lipschitz-free space $\mathcal{F}(M)$ over a compact metric space $M$ is a dual space if and only if $M$ is purely 1unrectifiable. Furthermore, for any complete metric space $M$, we deduce that pure 1-unrectifiability actually characterizes some well-known Banach space properties of $\mathcal{F}(M)$ such as the Radon-Nikodým property and the Schur property. A direct consequence is that any complete, purely 1-unrectifiable metric space isometrically embeds into a Banach space with the Radon-Nikodým property. Finally, we provide a solution to a problem of Whitney from 1935 by finding a rectifiability-based characterization of 1-critical compact metric spaces, and we use this characterization to prove the following: a bounded turning tree fails to be 1-critical if and only each of its subarcs has $\sigma$-finite Hausdorff 1-measure.
\end{abstract}

\section{INTRODUCTION}

A Lipschitz function $f: M \rightarrow \mathbb{R}$ on a metric space $(M, d)$ is said to be locally flat if

$$
\lim _{x, y \rightarrow p} \frac{|f(x)-f(y)|}{d(x, y)}=0
$$

for every $p \in M$. When $M$ is compact, the limit condition can be equivalently replaced by $d(x, y) \rightarrow 0$, uniformly in $p$. Locally flat Lipschitz functions appear throughout analysis - they are at the heart of Whitney's construction of a continuously differentiable function $\mathbb{R}^{2} \rightarrow \mathbb{R}$ that is nonconstant on a connected set of critical points [55], and they are deeply connected to functional analytic properties of the space of Lipschitz functions on $M$ ([52, Chapters 4 and 8]), to name some examples. In [50], Weaver isolated a property of locally flat Lipschitz functions that, for reasons soon to be clear, is now central to their study. Following [52, Defintion 4.10], we say that the locally flat Lipschitz functions separate points (of M) uniformly if there exists $C \in[1, \infty)$ such that for any $x, y \in M$, there exists a $C$-Lipschitz locally flat function $f: M \rightarrow \mathbb{R}$ with $f(x)-f(y)=d(x, y)$. The infimum of all such $C$ is called the separation constant. Despite the importance of this property, the exact conditions on $M$ ensuring its satisfaction or failure remained unclear. Indeed, Weaver writes in [51, p. 77-78], "Whether [the locally flat Lipschitz functions] separate points uniformly depends only on the metric space $M$, so it is disappointing that the condition cannot be formulated in a way that directly involves the geometry of $M$." The first main result of this article is a resolution of Weaver's

2020 Mathematics Subject Classification. Primary 51F30; Secondary 28A78, 30L05, 46B20, 46B22, 54E45.

Key words and phrases. Purely 1-unrectifiable, Radon-Nikodým property, Whitney arc, Lipschitz-free space, locally flat Lipschitz function. 
problem. Recall that a metric space $M$ is purely $k$-unrectifiable if, for every $A \subset \mathbb{R}^{k}$ and Lipschitz map $f: A \rightarrow M$, the Hausdorff $k$-measure of $f(A)$ equals 0 . By [39, Theorem 9], $M$ is purely 1-unrectifiable if and only if it contains no curve fragments - meaning bi-Lipschitz copies of compact, positive measure subsets of $\mathbb{R}$ (see also [19, Theorem 11.12]).

Theorem A. Let $M$ be a compact metric space. Then the locally flat Lipschitz functions on $M$ separate points uniformly if and only if $M$ is purely 1-unrectifiable.

Theorem A provides a geometric characterization of uniform separation of points by locally flat Lipschitz functions. From the opposite point-of-view, this theorem can also be seen as a functional characterization of purely 1-unrectifiable metric spaces. Our next theorem follows easily from Theorem A and standard results from $[52$, Chapter 4] and [38].

Theorem $\mathbf{A}^{\prime}$. Let $M$ be a compact metric space. Then $M$ is purely 1-unrectifiable if and only if the set of 1-Lipschitz locally flat functions on $M$ is dense in the set of all 1-Lipschitz functions on $M$ with respect to the sup-norm topology.

Theorem A' should be compared with Bate's version of the classical BesicovitchFederer theorem ([40, Theorem 18.1]), which yields a functional characterization of pure 1-unrectifiability under different hypotheses (see [40, Theorem 18.1] and [7, Theorem 1.1] for the statements of the respective theorems in full generality).

Bate's Besicovitch-Federer Theorem ([7, Theorem 1.1]). Let $M$ be a complete metric space with finite Hausdorff 1-measure. Then $M$ is purely 1-unrectifiable if and only if the set of 1-Lipschitz functions $f: M \rightarrow \mathbb{R}$ with $f(M)$ Lebesgue null is comeager in the set of all 1-Lipschitz functions on $M$ with respect to the sup-norm topology.

See Section 2 for the proof of Theorem A and for further discussion on the relationship between the present article and [7].

Our motivation to prove Theorem A comes from three corners of Lipschitz analysis: Lipschitz-free Banach spaces, bi-Lipschitz embeddings, and Whitney arcs. Partial results from each area indicated Theorem A was true, and with it in hand, we are able to complete several lines of research. We'll describe these in turn, beginning with a review of Lipschitz-free spaces.

For a metric space $(M, d)$ equipped with a distinguished point $0 \in M$, the Lipschitz-free space $\mathcal{F}(M)$ is a Banach space that is built around $M$ in such a way that $M$ is isometric to a subset $\delta(M)$ of $\mathcal{F}(M)$, and Lipschitz maps from $\delta(M)$ into any other Banach space $X$ uniquely extend to bounded linear operators from $\mathcal{F}(M)$ into $X$ (see Section 1.1 for a more detailed definition). In particular

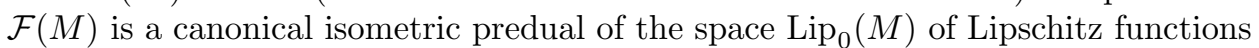
on $M$ vanishing at 0 endowed with the Lipschitz constant as a norm. Naturally, the study of Lipschitz-free spaces is at the intersection of functional analysis and metric geometry. Nevertheless, it is worth mentioning that Lipschitz-free spaces are studied in different research areas, for different reasons and under different names. For instance, thanks to the Kantorovich-Rubinstein duality theorem (e.g. [49, Theorem 1.14]), the norm on $\mathcal{F}(M)$ can be interpreted as the cost of the optimal solution of a certain transportation problem (see [52, Section 3.3], where $\mathcal{F}(M)$ is called Arens-Eells space). They are also of significant interest for computer science where the names that are commonly used for this distance are earth mover distance and transportation cost (e.g. [42]).

The first application of Theorem A is a characterization of Lipschitz-free spaces over compact spaces which are isometrically dual Banach spaces. The study of 
duality of Lipschitz-free spaces $\mathcal{F}(M)$ dates back to the 1960's. The first important result is due to Johnson [36], who proved that for any compact $M$ endowed with a snowflake metric (also known as a Hölder metric) the relation

$$
\mathcal{F}(M)=\operatorname{lip}_{0}(M)^{*}
$$

holds, where $\operatorname{lip}_{0}(M)$ is the "little Lipschitz space" consisting of all locally flat functions in $\operatorname{Lip}_{0}(M)$. On the other hand, Lebesgue's fundamental theorem of calculus easily implies $\operatorname{lip}_{0}([0,1])=\{0\}$, and so (1) cannot hold for $M=[0,1]$. More strongly, Godard proved in [27] that $\mathcal{F}(A)$ is isomorphic to $L_{1}$ whenever $A$ is a positive measure subset of $\mathbb{R}$, and therefore $\mathcal{F}(M)$ can never be a dual space when $M$ is separable but not purely 1-unrectifiable. Some time after Johnson's result, Weaver identified in [50] a powerful sufficient condition for (1): it is enough that $\operatorname{lip}_{0}(M)$ separates points of $M$ uniformly (a property enjoyed by snowflaked metric spaces $M$ ). Thus, Theorem A bridges the gap between the works of Weaver and Godard, and we arrive at our first major application.

Theorem B. Let $M$ be a compact metric space. Then the following are equivalent:

(i) $M$ is purely 1-unrectifiable,

(ii) $\mathcal{F}(M)$ is a dual space,

(iii) $\operatorname{lip}_{0}(M)$ is an isometric predual of $\mathcal{F}(M)$.

Theorem B unifies a series of results giving sufficient conditions under which $\mathcal{F}(M)$ is a dual space for compact $M[3,17,18,27,50]$ (see Section 3 for details), and in particular it solves the long-standing question regarding whether $\operatorname{lip}_{0}(M)$ must be one of its preduals in that case. We also show that, while $\operatorname{lip}_{0}(M)$ is never a unique predual of $\mathcal{F}(M)$ (unless $M$ is finite), it is the only predual that satisfies certain additional conditions (see Theorem 3.4). Section 3 contains the proof of Theorem B (through Theorem 3.1) and further discussion on duality of Lipschitz-free spaces.

In fact, when combined with previously known implications, Theorem B characterizes some well-known Banach space properties in Lipschitz-free spaces $\mathcal{F}(M)$ for compact $M$. These properties include the Radon-Nikodým property, the KreinMilman property and the Schur property (see Theorem 3.1 and the discussion thereafter). Recall that a Banach space $X$ has the Radon-Nikodým property (RNP) if every Lipschitz map $\mathbb{R} \rightarrow X$ is differentiable Lebesgue-almost everywhere; it has the Schur property if every weakly convergent sequence in $X$ is norm convergent; and it has the Krein-Milman property if every closed bounded convex set in $X$ is the closed convex hull of its extreme points.

This characterization can be generalized to noncompact $M$. It was recently shown in [2] that certain Banach space properties of Lipschitz-free spaces, including the Schur property, are compactly determined in the following sense: a Lipschitzfree space $\mathcal{F}(M)$ has the mentioned property if and only if the subspace $\mathcal{F}(K)$ has it for each compact $K \subset M$. This makes it possible to establish some results on Lipschitz-free spaces by reducing their proofs to the case where $M$ is compact. We prove here that the Radon-Nikodým property of Lipschitz-free spaces is also compactly determined (see Corollary 4.5), and so the next result follows from its compact version.

Theorem C. Let $M$ be a metric space. Then the following are equivalent:

(i) The completion of $M$ is purely 1-unrectifiable,

(ii) $\mathcal{F}(M)$ has the Radon-Nikodym property,

(iii) $\mathcal{F}(M)$ has the Krein-Milman property,

(iv) $\mathcal{F}(M)$ has the Schur property,

(v) $\mathcal{F}(M)$ contains no isomorphic copy of $L_{1}$. 
Theorem $\mathrm{C}$ is a culmination of many previous works that provided sufficient conditions for a Lipschitz-free space to have the Schur property and suggested that it would be equivalent to the Radon-Nikodým property [2, 26, 30, 38, 45] (see Section 4 for more information and for the proof of Theorem $C$ through Theorem 4.6). To the best of our knowledge, Theorem $\mathrm{C}$ is the first non-trivial characterization of isomorphic Banach space properties for $\mathcal{F}(M)$ in terms of metric properties of $M$ (see e.g. [3, 12, 25, 27, 48] for characterizations of some isometric properties). On top of that, it is a long-standing open problem in Banach Space theory whether the Radon-Nikodým and Krein-Milman properties are equivalent in general (see e.g. [23, p. 633]), and Theorem C solves it for the particular case of Lipschitz-free spaces. The equivalence of several other properties follows easily; see Remark 4.7 for a more detailed account.

Secondly, let us point out an application of the above results to bi-Lipschitz embedding theory. Banach spaces with the Radon-Nikodým property have gained popularity among metric space geometers because many well-known examples of metric spaces, such as the Heisenberg group or the Laakso space, fail to bi-Lipschitz embed into any one of them ([13, Theorem 1.6]). The two main methods used to prove non-bi-Lipschitz embeddability of metric spaces into Banach spaces with the RNP are due to Cheeger and Kleiner ([13, Theorem 1.6]) and Ostrovksii ([44, Theorem 1.3]). The common feature of these methods is that the metric spaces under consideration must possess a large collection of curve fragments (see [6] and the last paragraph starting on page 2 of [8]), and hence are far from being purely 1unrectifiable. The following theorem is a partial converse to the theories of CheegerKleiner and Ostrovskii. It is immediately implied by Theorem $\mathrm{C}$ and the fact that every metric space isometrically embeds into its Lipschitz-free space.

Theorem D. A complete, purely 1-unrectifiable metric space isometrically embeds into a Banach space with the Radon-Nikodym property.

Our final application is to the theory of Whitney sets, or 1-critical sets. In [55], Whitney constructed a $C^{1}$ function $f: \mathbb{R}^{2} \rightarrow \mathbb{R}$ such that $\nabla f$ vanishes on an arc $\gamma \subset \mathbb{R}^{2}$, but $f$ is not constant on $\gamma$. Following the terminology from [43], connected subsets $A$ of $\mathbb{R}^{n}$ for which there exists a $C^{1}$ function $f: \mathbb{R}^{n} \rightarrow \mathbb{R}$ that is nonconstant on $A$ and $\nabla f \equiv 0$ on $A$ are known as 1 -critical sets. These sets are very well-studied - for example, see $[14,16,31,43,53,55]$. By Whitney's extension theorem [54], a compact, connected set $A \subset \mathbb{R}^{n}$ is 1-critical if and only if it supports a nonconstant locally flat Lipschitz function, and thus 1-criticality can be defined as a purely metric notion in this way. Of course, if $M$ is rectifiably-connected, meaning every pair of points can be joined by a finite-length curve, then $M$ fails to be 1-critical. The obvious question is whether some sort of converse is true, and this problem was already posed in Whitney's original paper [55] where he wrote "it would be interesting to discover how far from rectifiable a closed set must be [to be 1-critical]" (see also [43, Q]). We provide a solution to Whitney's problem as application of Theorem A.

Theorem E. A compact metric space fails to be 1-critical if and only if it is transfinitely almost-rectifiably-connected.

See Section 5 for an explanation of transfinite almost-rectifiable-connectedness. Using Theorem E, we are able to prove a quantitative, measure-theoretic characterization of the 1-criticality property in bounded turning trees (see Definition 5.18), which includes the class of quasiarcs. 
Theorem F. Let $T$ be a 1-bounded turning tree, and for any $x, y \in T$, let $[x, y]$ denote the unique arc joining $x$ and $y$. For all $x, y \in T$,

$$
\sup _{f \in B_{\operatorname{lip}(T)}}|f(y)-f(x)|=\inf \left\{\mathcal{H}_{\infty}^{1}(A):[x, y] \backslash A \text { is } \mathcal{H}^{1}-\sigma \text {-finite }\right\}
$$

where $B_{\operatorname{lip}(T)}$ is the set of locally flat 1-Lipschitz functions. In particular, a bounded turning tree fails to be 1-critical if and only if each of its subarcs is $\mathcal{H}^{1}$ - $\sigma$-finite.

See Section 5 for the proofs of Theorems E (as Corollary 5.10) and F (as Theorem 5.20). Each one of the Theorems A - F is new even for metric spaces that are subsets of Euclidean space.

Notation. Throughout the paper, $M$ will stand for a metric space with metric $d$. We will assume without mention that $M$ is pointed, i.e. we have selected a distinguished point $0 \in M$. We will use the notation

$$
\begin{aligned}
d(p, A) & =\inf \{d(p, x): x \in A\} \\
{[A]_{r} } & =\{x \in M: d(x, A) \leq r\} \\
B_{r}(p) & =\{x \in M: d(x, p) \leq r\} \\
\operatorname{rad}(A) & =\sup \{d(x, 0): x \in A\} \\
\operatorname{diam}(A) & =\sup \{d(x, y): x, y \in A\}
\end{aligned}
$$

for $p \in M, A \subset M$ and $r \geq 0$. For convenience of the reader, let us recall the vector spaces

$$
\begin{aligned}
\operatorname{Lip}(M) & =\left\{f \in \mathbb{R}^{M}: f \text { Lipschitz }\right\} \\
\operatorname{lip}(M) & =\{f \in \operatorname{Lip}(M): f \text { locally flat }\} \\
\operatorname{Lip}_{0}(M) & =\{f \in \operatorname{Lip}(M): f(0)=0\} \\
\operatorname{lip}_{0}(M) & =\{f \in \operatorname{lip}(M): f(0)=0\} .
\end{aligned}
$$

Whenever $V$ is one of the four spaces above, we have the unit ball

$$
B_{V}=\{f \in V: f \text { is 1-Lipschitz }\} .
$$

Note that the meaning of $\operatorname{lip}(M)$ for noncompact $M$ is not entirely consistent throughout the existing literature. For instance, some authors (e.g. [38]) have used $\operatorname{lip}_{0}(M)$ to denote the Lipschitz functions $f: M \rightarrow \mathbb{R}$ satisfying the uniform local flatness property

$$
\lim _{d(x, y) \rightarrow 0} \frac{|f(x)-f(y)|}{d(x, y)}=0 .
$$

When $M$ is not compact, this definition is more restrictive than the one we give, but this is of little consequence since our main results concerning $\operatorname{lip}_{0}(M)$ are stated only for compact spaces (except for a short departure into proper spaces in Section 3).

We use the lattice-theoretic notation $f \vee g$ and $f \wedge g$ for the pointwise maximum and minimum of the functions (or constants) $f$ and $g$, respectively. It holds that $f \vee g, f \wedge g \in B_{\operatorname{Lip}(M)}$ whenever $f, g \in B_{\operatorname{Lip}(M)}$, and similarly for $B_{\operatorname{lip}(M)}$.

The Lebesgue measure on $\mathbb{R}$ will be denoted by $\lambda$.

1.1. Preliminaries on Lipschitz spaces and Lipschitz-free spaces. For a metric space $M$ with a distinguished base point $0 \in M$, the Lipschitz-free space or Arens-Eells space $\mathcal{F}(M)$ is a Banach space constructed around $M$ which is characterized by the following property: any Banach space-valued Lipschitz map $f: M \rightarrow X$ vanishing at 0 can be extended in a unique way to a continuous linear map $\widehat{f}: \mathcal{F}(M) \rightarrow X$ whose operator norm is equal to the best Lipschitz constant of $f$. This is often referred to as the "universal extension property" of Lipschitz-free spaces. 
There are several ways to construct the Lipschitz-free space over $M$ (see e.g. [15, Chapter 8]). We will focus on the following one: consider the Banach space $\operatorname{Lip}_{0}(M)$ equipped with the norm

$$
\|f\|_{L}=\sup \left\{\frac{f(x)-f(y)}{d(x, y)}: x \neq y \in M\right\}
$$

(note that $\|\cdot\|_{L}$ is not a norm on $\operatorname{Lip}(M)$, and it is for this reason that we work with $\operatorname{Lip}_{0}(M)$ instead). There are evaluation functionals $\delta(x) \in \operatorname{Lip}_{0}(M)^{*}$ given by $\delta(x): f \mapsto f(x)$ for $x \in M$, and $\mathcal{F}(M)$ can be realized as the norm-closed linear span of $\{\delta(x): x \in M\}$ in the Banach space $\operatorname{Lip}_{0}(M)^{*}$. An easy consequence of the universal extension property (taking $X=\mathbb{R}$ ) is that $\mathcal{F}(M)$ is an isometric predual of $\operatorname{Lip}_{0}(M)$, and the corresponding weak* topology on $B_{\operatorname{Lip}_{0}(M)}$ coincides with the topology of pointwise convergence. When $M$ is compact, it also agrees with the topology of uniform convergence. Another consequence is that, for any subset $M^{\prime} \subset M, \mathcal{F}\left(M^{\prime}\right)$ may be canonically identified with the closed subspace of $\mathcal{F}(M)$ generated by the evaluation functionals on points of $M^{\prime}$. These facts will be used repeatedly in the sequel.

The set $\operatorname{lip}_{0}(M)$ is a norm-closed subspace of $\operatorname{Lip}_{0}(M)$, and generally it is not weak*-closed. Another fact we will use implicitly is that $g \circ f \in \operatorname{lip}(M)$ whenever $f: M \rightarrow N$ is Lipschitz and $g \in \operatorname{lip}(N)$.

We refer the reader to [52] for basic properties of Lipschitz-free spaces and to [28] for a survey on their applications to the nonlinear geometry of Banach spaces.

1.2. Preliminaries on Hausdorff Measure and Hausdorff Convergence. We review the basic properties of Hausdorff measure and content and Hausdorff metric and convergence. For more details, we refer the reader to [40] and [35].

Definition 1.1 (Hausdorff Measure and Content). For $M$ a separable metric space and $\delta \in(0, \infty]$, define

$$
\mathcal{H}_{\delta}^{1}(M):=\inf \left\{\sum_{i=1}^{\infty} \operatorname{diam}\left(E_{i}\right): M \subset \bigcup_{i=1}^{\infty} E_{i}, \operatorname{diam}\left(E_{i}\right)<\delta\right\},
$$

and

$$
\mathcal{H}^{1}(M):=\lim _{\delta \rightarrow 0} \mathcal{H}_{\delta}^{1}(M)
$$

$\mathcal{H}^{1}(M)$ is called the Hausdorff 1-measure of $M$ and $\mathcal{H}_{\infty}^{1}(M)$ its Hausdorff 1-content.

Remark 1.2. By replacing each $E_{i}$ by $\left[E_{i}\right]_{\varepsilon_{i}}$ with suitable $\varepsilon_{i}>0$, we see that the definition of $\mathcal{H}_{\delta}^{1}(M)$ is unchanged if we require that the interiors of the sets $E_{i}$ cover $M$. Hence if $M$ is compact, the definition of $\mathcal{H}_{\delta}^{1}(M)$ is unchanged if we require the cover $\left\{E_{i}\right\}_{i}$ to be finite instead of countably infinite. We use this well-known fact in Lemma 1.5 and in Section 2.

As the name suggests, Hausdorff 1-measure is a Borel measure on any metric space. While $\mathcal{H}_{\delta}^{1}$ is in general not a measure for $\delta>0$, it is $\sigma$-subadditive. Clearly, it always holds that $\mathcal{H}^{1}(M) \geq \mathcal{H}_{\infty}^{1}(M)$. The reverse inequality is not true in general, but it clearly does hold that $\mathcal{H}_{\infty}^{1}(M)=0 \Longleftrightarrow \mathcal{H}^{1}(M)=0$. We use this fact in Section 5.

Definition 1.3. For $(\Omega, d)$ a compact metric space, the space of compact subsets of $\Omega$ is called the hyperspace of $\Omega$. It is itself a compact metric space (see [35, Theorems 3.1 and 3.5] with respect to the Hausdorff metric defined by

$$
d_{H}\left(K_{1}, K_{2}\right):=\inf \left\{\varepsilon>0: K_{1} \subset\left[K_{2}\right]_{\varepsilon}, K_{2} \subset\left[K_{1}\right]_{\varepsilon}\right\} .
$$

Remark 1.4. It is easy to verify that whenever $X$ is a compact metric space, $K_{j}, K \subset$ $\Omega$ are compact with $K_{j} \rightarrow K$ with respect to the Hausdorff metric, and $f_{j}, f: \Omega \rightarrow$ 
$X$ are continuous with $f_{j} \rightarrow f$ uniformly, then $f_{j}\left(K_{j}\right) \rightarrow f(K)$ with respect to the Hausdorff metric on the hyperspace of $X$.

An important feature of Hausdorff content is its upper semi-continuity with respect to Hausdorff convergence, a feature not enjoyed by Hausdorff measure.

Lemma 1.5 (Upper Semi-Continuity of Hausdorff Content). Let $(\Omega, d)$ be a compact metric space. Suppose $K_{j} \rightarrow K$ with respect to the Hausdorff metric on the hyperspace of $\Omega$. Then

$$
\mathcal{H}_{\infty}^{1}(K) \geq \limsup _{j \rightarrow \infty} \mathcal{H}_{\infty}^{1}\left(K_{j}\right) .
$$

Proof. Let $\varepsilon>0$ be arbitrary. Choose $E_{1}, \ldots, E_{n} \subset \Omega$ such that $K \subset \bigcup_{i=1}^{n} E_{i}$ and $\mathcal{H}_{\infty}^{1}(K)+\varepsilon \geq \sum_{i=1}^{n} \operatorname{diam}\left(E_{i}\right)$. Since $K_{j} \rightarrow K$, there is $J \in \mathbb{N}$ so that $K_{j} \subset[K]_{\varepsilon / n}$ for all $j \geq J$. Then $K_{j} \subset \bigcup_{i=1}^{n}\left[E_{i}\right]_{\varepsilon / n}$, and hence

$$
\begin{aligned}
\limsup _{j \rightarrow \infty} \mathcal{H}_{\infty}^{1}\left(K_{j}\right)=\inf _{J \in \mathbb{N}} \sup _{j \geq J} \mathcal{H}_{\infty}^{1}\left(K_{j}\right) & \leq \sum_{i=1}^{n} \operatorname{diam}\left(\left[E_{i}\right]_{\varepsilon / n}\right) \\
& \leq 2 \varepsilon+\sum_{i=1}^{n} \operatorname{diam}\left(E_{i}\right) \leq 3 \varepsilon+\mathcal{H}_{\infty}^{1}(K) .
\end{aligned}
$$

Since $\varepsilon>0$ was arbitrary, the conclusion follows.

\section{Proof of Theorem A}

We recall Theorem A and then discuss its proof.

Theorem A. Let $M$ be a compact metric space. Then the locally flat Lipschitz functions on $M$ separate points uniformly if and only if $M$ is purely 1-unrectifiable.

Let us first remark that the proof of the "only if" implication is easy and wellknown. Indeed, if $K$ bi-Lipschitz embeds into $M$ and $\operatorname{lip}(M)$ separates the points of $M$ uniformly, then lip $(K)$ separates the points of $K$ uniformly. Using Lebesgue's density theorem and fundamental theorem of calculus, it is not difficult to check that, whenever $K \subset \mathbb{R}$ is compact with positive measure, $\operatorname{lip}(K)$ does not separate the points of $K$ uniformly (see [52, Example 4.13(b)] for details). Consequently, $\operatorname{lip}(M)$ cannot separate the points of $M$ uniformly if $M$ contains a curve fragment $\gamma(K)$. In fact, this argument works when $M$ is any metric space; compactness isn't necessary. The real content of Theorem A is the "if" direction, which is implied by the following seemingly stronger statement.

Theorem 2.1. If $M$ is compact and purely 1-unrectifiable then, for all $p \in M$ and $\delta>0$, there exists $g \in B_{\operatorname{lip}(M)}$ such that $g(x)-g(p) \geq d(p, x)-\delta$ for every $x \in M$.

We use the remainder of this section to prove Theorem 2.1. The proof occurs in the last subsection, following a host of supporting lemmas. First, we recall a useful method for constructing Lipschitz functions on a metric space $M$ with prescribed local behavior. We describe the method here and summarize the conclusion in Proposition 2.4. The method requires $M$ to be isometrically embedded in a convex subset $\Omega$ of a Banach space (actually, a geodesic metric space would suffice). However, there is no loss of generality in making this assumption, because every metric space $N$ isometrically embeds into the Lipschitz-free space $\mathcal{F}(N)$. Additionally, when $M$ is compact, there is no loss of generality in assuming that $\Omega$ is also compact, because closed convex hulls of compact subsets of Banach spaces are compact. Before stating the construction, we briefly review the definition of path integrals and length measure for the purpose of setting notation. 
Definition 2.2 (Length Measure and Path Integrals). Let $(\Omega, d)$ be a metric space. When $\gamma:[a, b] \rightarrow \Omega$ is a Lipschitz curve, we get a total variation measure $T V_{\gamma}$ on $[a, b]$ defined on intervals by

$$
T V_{\gamma}([s, t]):=\sup \left\{\sum_{i=1}^{n} d\left(\gamma\left(t_{i-1}\right), \gamma\left(t_{i}\right)\right): s=t_{0}<t_{1}<\cdots<t_{n}=t\right\} \text {. }
$$

Since $\gamma$ is Lipschitz, $T V_{\gamma} \leq L \cdot \lambda$ for some $L<\infty$. Pushing forward the measure $T V_{\gamma}$ under $\gamma$ gives a finite, positive Borel measure $\mu_{\gamma}$ on $\Omega$, called the length measure of $\gamma$. The length measure is invariant with respect to Lipschitz reparametrizations. When $f: \Omega \rightarrow \mathbb{R}$ is bounded Borel, we get a path integral defined by

$$
\int_{\gamma} f d s:=\int_{\Omega} f d \mu_{\gamma}=\int_{a}^{b}(f \circ \gamma) d T V_{\gamma} .
$$

The length of $\gamma$ is $|\gamma|:=\int_{\gamma} 1 d s=\mu_{\gamma}(\Omega)=T V_{\gamma}([a, b])$.

We are now ready to state our main method of constructing locally flat Lipschitz functions.

Definition 2.3. Given a convex subset $\Omega \subset X$ of a Banach space $X, p \in \Omega$, and a bounded Borel function $f: \Omega \rightarrow[0, \infty)$, we define a function $\phi_{f, p}: \Omega \rightarrow[0, \infty)$ by

$$
\phi_{f, p}(x):=\inf _{\gamma} \int_{\gamma} f d s,
$$

for $x \in \Omega$, where the infimum is over all $a \leq b \in \mathbb{R}$ and Lipschitz curves $\gamma:[a, b] \rightarrow \Omega$ with $\gamma(a)=p$ and $\gamma(b)=x$.

The function $\phi_{f, p}$ should be thought as an "antiderivative" of $f$ of sorts. Note that $\phi_{f, p}(p)$ vanishes at $p$. Let us quickly check that $\phi_{f, p}$ is Lipschitz. Let $x, y \in \Omega$. Without loss of generality, we may assume $\phi_{f, p}(x) \leq \phi_{f, p}(y)$. Let $\varepsilon>0$, and choose $\gamma:[a, b] \rightarrow \Omega$ with $\gamma(a)=p, \gamma(b)=x$, and $\int_{\gamma} f d s<\phi_{f, p}(x)+\varepsilon$. Let

$$
[x, y]:=\{(1-t) x+t y \in X: t \in[0,1]\}
$$

denote the line segment in $X$ connecting $x$ and $y$. Since $x, y \in \Omega$ and $\Omega$ is convex, $[x, y] \subset \Omega$. Let $\gamma_{[x, y]}:[b, b+\|x-y\|] \rightarrow[x, y]$ denote the unit speed parametrization starting at $x$ and ending at $y$. Then we create a new Lipschitz curve $\tilde{\gamma}:[a, b+\|x-y\|] \rightarrow \Omega$ by concatenating $\gamma$ with $\gamma_{[x, y]}$. Specifically,

$$
\tilde{\gamma}(t):=\left\{\begin{array}{ll}
\gamma(t) & , \text { if } t \in[a, b] \\
\gamma_{[x, y]}(t) & , \text { if } t \in[b, b+\|x-y\|]
\end{array} .\right.
$$

It is easy to see that $\tilde{\gamma}$ is Lipschitz, $\tilde{\gamma}(a)=p$, and $\tilde{\gamma}(b+\|x-y\|)=y$. Thus, $\phi_{f, p}(y) \leq \int_{\tilde{\gamma}} f d s$. Then we have

$$
\begin{aligned}
\left|\phi_{f, p}(y)-\phi_{f, p}(x)\right| & =\phi_{f, p}(y)-\phi_{f, p}(x)<\int_{\tilde{\gamma}} f d s-\int_{\gamma} f d s+\varepsilon \\
& =\int_{\gamma_{[x, y]}} f d s+\varepsilon \leq \int_{\gamma_{[x, y]}}\|f\|_{L_{\infty}([x, y])} d s+\varepsilon \\
& =\|f\|_{L_{\infty}([x, y])}\left|\gamma_{[x, y]}\right|+\varepsilon=\|f\|_{L_{\infty}([x, y])}\|x-y\|+\varepsilon .
\end{aligned}
$$

Since $\varepsilon>0$ was arbitrary, this shows

$$
\left|\phi_{f, p}(y)-\phi_{f, p}(x)\right| \leq\|f\|_{L_{\infty}([x, y])}\|x-y\| .
$$

This inequality easily implies the following proposition. 
Proposition 2.4. Let $\Omega$ be a convex subset of a Banach space. For all $M \subset \Omega$, $p \in \Omega$, and $f: \Omega \rightarrow[0,1]$ Borel with $\lim _{r \rightarrow 0} \sup _{x \in[M]_{r}} f(x)=0$, we have $\phi_{f, p}(p)=0$ and $\phi_{f, p} \uparrow_{M} \in B_{\operatorname{lip}(M)}$.

A variation of this construction was used by Bate in [7, Section 3], providing us an invaluable starting point. In [7, Lemma 3.4], he proved that, for every compact purely 1-unrectifiable metric space $M$ and $\varepsilon>0$, the set

$$
B_{\operatorname{lip}^{\varepsilon}(M)}:=\left\{f \in B_{\operatorname{Lip}(M)}: \limsup _{d(x, y) \rightarrow 0} \frac{|f(x)-f(y)|}{d(x, y)} \leq \varepsilon\right\}
$$

is sup-norm dense in $B_{\operatorname{Lip}(M)}$. Even though it holds that $B_{\operatorname{lip}(M)}=\bigcap_{\varepsilon>0} B_{\operatorname{lip} \varepsilon}(M)$, it cannot be deduced by any typical mean that $B_{\operatorname{lip}(M)}$ is sup-norm dense in $B_{\operatorname{Lip}(M)}$, and fundamental compactness properties break down when working with $B_{\operatorname{lip}(M)}$ in place of $B_{\operatorname{lip}^{\varepsilon}(M)}$. Thus new arguments are required to prove Theorem 2.1.

2.1. Constructing the Separating Locally Flat Lipschitz Function. The next lemma is the linchpin of this section. We crucially use the compactness of $\Omega$ in its proof. It is also the only time we directly appeal to the pure 1-unrectifiability of $M$.

Lemma 2.5 (Neighborhood Inducing Small Hausdorff Content). For every compact metric space $\Omega$, purely 1-unrectifiable closed subset $M \subset \Omega, L<\infty$, and $\varepsilon>0$, there exists a compact neighborhood $V$ of $M$ in $\Omega$ such that $\mathcal{H}_{\infty}^{1}(\operatorname{im}(\gamma) \cap V)<\varepsilon$ for every Lipschitz curve $\gamma:[a, b] \rightarrow \Omega$ with $|\gamma| \leq L$.

Proof. Let $\Omega$ and $M$ be as above, and suppose the lemma is false. Then we can find $L<\infty, \varepsilon>0$, and Lipschitz curves $\gamma_{n}:\left[a_{n}, b_{n}\right] \rightarrow \Omega$ with $\left|\gamma_{n}\right| \leq L$ and $\mathcal{H}_{\infty}^{1}\left(\operatorname{im}\left(\gamma_{n}\right) \cap[M]_{1 / n}\right) \geq \varepsilon$ for every $n$. By parametrizing by arclength on $\left[0,\left|\gamma_{n}\right|\right]$ and constant on $\left[\left|\gamma_{n}\right|, L\right]$, we may assume each $\gamma_{n}:[0, L] \rightarrow \Omega$ is 1-Lipschitz. Set $K_{n}:=\gamma_{n}^{-1}\left([M]_{1 / n}\right) \subset[0, L]$, so that $\gamma_{n}\left(K_{n}\right)=\operatorname{im}\left(\gamma_{n}\right) \cap[M]_{1 / n}$. By the ArzelàAscoli theorem, we may assume $\gamma_{n}$ converges to some 1-Lipschitz $\gamma:[0, L] \rightarrow \Omega$ uniformly. By compactness of the hyperspace of $[0, L]$, we may also assume that $K_{n} \rightarrow K$ with respect to the Hausdorff metric for some compact $K \subset[0, L]$. It follows that $\gamma_{n}\left(K_{n}\right) \rightarrow \gamma(K)$ with respect to the Hausdorff metric on the hyperspace of $\Omega$. Lemma 1.5 implies $\mathcal{H}_{\infty}^{1}(\gamma(K)) \geq \varepsilon$. It also holds that $\gamma(K) \subset M$. To see this, let $\eta>0$ be arbitrary. Choose $n$ large enough so that $d_{H}\left(\gamma_{n}\left(K_{n}\right), \gamma(K)\right)<\eta$ and $1 / n<\eta$. Then $\gamma(K) \subset\left[\gamma_{n}\left(K_{n}\right)\right]_{\eta}$ and $\gamma_{n}\left(K_{n}\right) \subset[M]_{\eta}$, hence $\gamma(K) \subset[M]_{2 \eta}$. Since $M$ is closed and $\eta>0$ was arbitrary, it follows that $\gamma(K) \subset M$. Thus, $\gamma:[0, L] \rightarrow \Omega$ is Lipschitz and

$$
\mathcal{H}^{1}(\operatorname{im}(\gamma) \cap M) \geq \mathcal{H}^{1}(\gamma(K)) \geq \mathcal{H}_{\infty}^{1}(\gamma(K)) \geq \varepsilon>0,
$$

contradicting pure 1-unrectifiability of $M$.

Throughout the remainder of this subsection, let $\Omega$ be a compact, convex subset of a Banach space, with induced metric denoted $d$. Let $M$ be a closed, purely 1-unrectifiable subset of $\Omega$ and let $\delta>0$. Let $M \subset \ldots V_{2} \subset V_{1} \subset V_{0}=\Omega$ be a decreasing sequence of compact neighborhoods of $M$ such that, for all $n \geq 0$ and Lipschitz curves $\gamma:[a, b] \rightarrow \Omega$,

$$
|\gamma| \leq n \Rightarrow \mathcal{H}_{\infty}^{1}\left(\operatorname{im}(\gamma) \cap V_{n}\right)<\delta 2^{-n} .
$$

Such a sequence exists by Lemma 2.5 .

In the proof of Theorem 2.1, we will use these neighborhoods to construct a bounded Borel function $f: \Omega \rightarrow[0,1]$ that is constant on $V_{n-1} \backslash V_{n}$, and the corresponding Lipschitz function $\phi_{f, p}$ will be used to fulfill Theorem 2.1. To obtain 
the necessary estimates on $\phi_{f, p}$, we need three lemmas about modifying Lipschitz curves. Each lemma builds off the previous one.

Lemma 2.6 (Curve Modification in Set of Small Diameter). For any Lipschitz curve $\gamma:[a, b] \rightarrow \Omega, r \geq 0$, and compact, convex subset $E \subset \Omega$ of diameter $r$, there exists another Lipschitz curve $\tilde{\gamma}:[a, b] \rightarrow \Omega$ such that

- $\tilde{\gamma}$ has the same endpoint values as $\gamma$,

- $\mu_{\tilde{\gamma}}(E) \leq r$, and

- for every Borel $A \subset \Omega, \mu_{\tilde{\gamma}}(A \backslash E) \leq \mu_{\gamma}(A \backslash E)$.

Consequently, $\mu_{\tilde{\gamma}} \leq \mu_{\gamma}+r$.

Proof. Let $\gamma, r, E$ be as above. If $\operatorname{im}(\gamma) \cap E=\emptyset$, we simply choose $\tilde{\gamma}=\gamma$ and the proof is finished. So assume $\operatorname{im}(\gamma) \cap E \neq \emptyset$. Let

$$
\begin{aligned}
& s_{0}:=\min \{t \in[a, b]: \gamma(t) \in E\} \\
& s_{1}:=\max \{t \in[a, b]: \gamma(t) \in E\}
\end{aligned}
$$

which exist by compactness of $E$ and continuity of $\gamma$. Define $\tilde{\gamma}$ on $\left[a, s_{0}\right] \cup\left[s_{1}, b\right]$ to agree with $\gamma$, and on $\left[s_{0}, s_{1}\right]$ to be the constant speed parametrization of the line segment between $\gamma\left(s_{0}\right)$ and $\gamma\left(s_{1}\right)$. This line segment belongs to $E$ by convexity, and $\tilde{\gamma}$ satisfies the desired properties.

Lemma 2.7 (Curve Modification in Set of Small Hausdorff Content). For any Lipschitz curve $\gamma:[a, b] \rightarrow \Omega, \varepsilon>0$, and compact $K \subset \Omega$ with $\mathcal{H}_{\infty}^{1}(\operatorname{im}(\gamma) \cap K)<\varepsilon$, there exists another Lipschitz curve $\tilde{\gamma}:[a, b] \rightarrow \Omega$ such that

- $\tilde{\gamma}$ has the same endpoint values as $\gamma$,

- $\mu_{\tilde{\gamma}}(K)<\varepsilon$, and

- $\mu_{\tilde{\gamma}}<\mu_{\gamma}+\varepsilon$.

Proof. Let $\gamma, \varepsilon, K$ be as above. Since $\mathcal{H}_{\infty}^{1}(\operatorname{im}(\gamma) \cap K)<\varepsilon$, there exist finitely many subsets $E_{1}, \ldots E_{n} \subset \Omega$ of diameters $r_{1}, \ldots r_{n} \geq 0$ such that $\operatorname{im}(\gamma) \cap K \subset \bigcup_{i=1}^{n} E_{i}$ and $\sum_{i=1}^{n} r_{i}<\varepsilon$. Since the diameter of $E_{i}$ is the same as that of its closed convex hull, we may assume each $E_{i}$ is compact and convex. Apply Lemma 2.6 with $\gamma=\gamma$, $r=r_{1}$, and $E=E_{1}$ to obtain a Lipschitz curve $\gamma_{1}:[a, b] \rightarrow \Omega$ satisfying

- $\gamma_{1}$ has the same endpoint values as $\gamma$,

- $\mu_{\gamma_{1}}\left(E_{1}\right) \leq r_{1}$, and

- $\mu_{\gamma_{1}} \leq \mu_{\gamma}+r_{1}$.

Then we apply Lemma 2.6 again with $\gamma=\gamma_{1}, r=r_{2}$, and $E=E_{2}$ to obtain a Lipschitz curve $\gamma_{2}:[a, b] \rightarrow \Omega$ satisfying

- $\gamma_{2}$ has the same endpoint values as $\gamma_{1}$, which are the same as $\gamma^{\prime}$ 's,

- $\mu_{\gamma_{2}}\left(E_{1} \cup E_{2}\right)=\mu_{\gamma_{2}}\left(E_{1} \backslash E_{2}\right)+\mu_{\gamma_{2}}\left(E_{2}\right)$

$\leq \mu_{\gamma_{1}}\left(E_{1} \backslash E_{2}\right)+r_{2} \leq r_{1}+r_{2}$, and

- $\mu_{\gamma_{2}} \leq \mu_{\gamma_{1}}+r_{2} \leq \mu_{\gamma}+r_{1}+r_{2}$.

Repeating this up to $n$ times produces a Lipschitz curve $\gamma_{n}:[a, b] \rightarrow \Omega$ satisfying

- $\gamma_{n}$ has the same endpoint values as $\gamma$,

- $\mu_{\gamma_{n}}\left(\bigcup_{i=1}^{n} E_{i}\right) \leq \sum_{i=1}^{n} r_{i}$, and

- $\mu_{\gamma_{n}} \leq \mu_{\gamma}+\sum_{i=1}^{n} r_{i}$.

Since $\operatorname{im}\left(\gamma_{n}\right) \cap K \subset \bigcup_{i=1}^{n} E_{i}$ and $\sum_{i=1}^{n} r_{i}<\varepsilon, \tilde{\gamma}=\gamma_{n}$ satisfies the desired properties.

Lemma 2.8 (Curve Modification in $V_{n}$ ). For any Lipschitz curve $\gamma:[a, b] \rightarrow \Omega$ with $\gamma(a) \neq \gamma(b)$, there exist another Lipschitz curve $\tilde{\gamma}:[a, b] \rightarrow \Omega$ and $m \in \mathbb{N}$ such that

- $\tilde{\gamma}$ has the same endpoint values as $\gamma$, 
- $m-1<|\tilde{\gamma}|$,

- $\mu_{\tilde{\gamma}}\left(V_{m}\right)<\delta$, and

- $\mu_{\tilde{\gamma}}<\mu_{\gamma}+\delta$.

Consequently, for every Borel function $f: \Omega \rightarrow[0,1]$ we have

$$
\int_{\gamma} f d s \geq \int_{\tilde{\gamma}} f d s-\delta \geq|\tilde{\gamma}| \inf _{\Omega \backslash V_{m}} f-2 \delta .
$$

Proof. Let $\gamma$ be as above. Let $A$ be the set of all $n \in \mathbb{N}$ such that there exists a Lipschitz curve $\tilde{\gamma}:[a, b] \rightarrow \Omega$ satisfying

- $\tilde{\gamma}$ has the same endpoint values as $\gamma$,

- $\mu_{\tilde{\gamma}}\left(V_{n}\right)<\delta$, and

- $\mu_{\tilde{\gamma}}<\mu_{\gamma}+\delta \sum_{i=n}^{\infty} 2^{-i}$.

Let $n:=\lceil|\gamma|\rceil$ be the least integer greater than or equal to $|\gamma|$. Note that $n \geq 1$ since $\gamma(a) \neq \gamma(b)$. The definition (2) of $V_{n}$ implies $\mathcal{H}_{\infty}^{1}\left(\operatorname{im}(\gamma) \cap V_{n}\right)<\delta 2^{-n}$. Then we apply Lemma 2.7 with $\gamma=\gamma, \varepsilon=\delta 2^{-n}$, and $K=V_{n}$ to obtain a Lipschitz curve $[a, b] \rightarrow \Omega$ that witnesses $n \in A$. Thus $A \neq \emptyset$, and $m:=\min (A)$ exists. Let $\tilde{\gamma}$ be a Lipschitz curve witnessing $m \in A$. We will show it must hold that $m-1<|\tilde{\gamma}|$, which will finish the proof.

Since $\tilde{\gamma}$ has the same endpoint values as $\gamma$ and $\gamma(a) \neq \gamma(b)$, it holds that $|\tilde{\gamma}|>0$. Hence, we are done if $m-1=0$. In then remaining case, when $m-1 \geq 1$, we assume towards a contradiction that $|\tilde{\gamma}| \leq m-1$. Then by definition of $V_{m-1}$, $\mathcal{H}_{\infty}^{1}\left(\operatorname{im}(\tilde{\gamma}) \cap V_{m-1}\right)<\delta 2^{-m+1}$. Then we apply Lemma 2.7 with $\gamma=\tilde{\gamma}, \varepsilon=\delta 2^{-m+1}$, and $K=V_{m-1}$ to obtain a Lipschitz curve $\gamma_{0}:[a, b] \rightarrow \Omega$ satisfying

- $\gamma_{0}$ has the same endpoint values as $\tilde{\gamma}$, which are the same as $\gamma$ 's,

- $\mu_{\gamma_{0}}\left(V_{m-1}\right)<\delta$, and

- $\mu_{\gamma_{0}}<\mu_{\tilde{\gamma}}+\delta 2^{-m+1}<\mu_{\gamma}+\delta \sum_{i=m}^{\infty} 2^{-i}+\delta 2^{-m+1}=\mu_{\gamma}+\delta \sum_{i=m-1}^{\infty} 2^{-i}$.

Thus, $\gamma_{0}$ witnesses $m-1 \in A$ (together with our prior assumption that $m-1 \geq 1$ ). This contradicts $m=\min (A)$.

To prove the last part of the lemma, notice that

$$
\int_{\tilde{\gamma}} f d s=\int_{\Omega} f d \mu_{\tilde{\gamma}} \leq \int_{\Omega} f d \mu_{\gamma}+\delta\|f\|_{\infty} \leq \int_{\gamma} f d s+\delta .
$$

Now, since $\mu_{\tilde{\gamma}}\left(V_{m}\right)<\delta$, we have

$$
\int_{\tilde{\gamma}} 1_{\Omega \backslash V_{m}} d s \geq|\tilde{\gamma}|-\delta .
$$

Thus, by positivity of $f$, we obtain

$$
\int_{\tilde{\gamma}} f d s \geq(|\tilde{\gamma}|-\delta) \inf _{\Omega \backslash V_{m}} f \geq|\tilde{\gamma}| \inf _{\Omega \backslash V_{m}} f-\delta .
$$

We are now prepared to describe the construction of the appropriate bounded Borel function $f: \Omega \rightarrow[0,1]$ and use Lemma 2.5 to get necessary estimates on $\inf _{\gamma} \int_{\gamma} f d s$.

\subsection{Proof of Theorem 2.1.}

Proof of Theorem 2.1. Let $p \in M$ and $\delta>0$. Set $c_{0}=1$ and

$$
c_{n}:=1 \wedge \frac{\operatorname{diam}(\Omega)}{n}
$$

for $n \geq 1$. Define the Borel function $f: \Omega \rightarrow[0,1]$ by

$$
f:= \begin{cases}c_{n} & \text { on } V_{n} \backslash V_{n+1} \\ 0 & \text { on } \bigcap_{n=0}^{\infty} V_{n} .\end{cases}
$$


Then since $c_{n} \searrow 0, \lim _{r \rightarrow 0} \sup _{x \in[M]_{r}} f(x)=0$. Hence, Proposition 2.4 implies $g:=$ $\phi_{f, p} \uparrow_{M} \in B_{\operatorname{lip}(M)}$.

Let $x \in \Omega$ and $\gamma:[a, b] \rightarrow \Omega$ a Lipschitz curve such that $\gamma(a)=p, \gamma(b)=x$. Then by Lemma 2.8, there is a Lipschitz curve $\tilde{\gamma}:[a, b] \rightarrow \Omega$ and $n \geq 0$ (indeed, take $n=m-1)$ such that $\tilde{\gamma}(a)=p, \tilde{\gamma}(b)=x, n \leq|\tilde{\gamma}|$, and

$$
\int_{\gamma} f d s \geq c_{n}|\tilde{\gamma}|-2 \delta
$$

Since $\tilde{\gamma}$ connects $p$ to $x$, it must hold that $d(p, x) \leq|\tilde{\gamma}|$, and thus

$$
c_{n}|\tilde{\gamma}| \geq c_{n} d(p, x) \vee c_{n} n .
$$

If $\operatorname{diam}(\Omega) \geq n$, then $c_{n} d(p, x)=d(p, x)$, and if $\operatorname{diam}(\Omega) \leq n$, then $c_{n} n=$ $\operatorname{diam}(\Omega) \geq d(p, x)$. Then, in all cases,

$$
c_{n}|\tilde{\gamma}| \geq d(p, x) .
$$

This inequality, together with (3), the definition of $g$ and the fact that $\gamma$ was arbitrary yield

$$
g(x)-g(p) \geq d(p, x)-2 \delta,
$$

which we wanted to prove.

\section{DuAlity of LipschitZ-FREe SPACES}

We will now use the results from Section 2 to obtain a characterization of several Banach space properties in Lipschitz-free spaces over compact spaces. One of the goals is to characterize the compact metric spaces $M$ such that the Lipschitz-free space $\mathcal{F}(M)$ is a dual. Not all compacts satisfy this; for instance, $\mathcal{F}([0,1])=L_{1}$ is not a dual space. For all previously known examples where $\mathcal{F}(M)$ is a dual, we actually have

$$
\mathcal{F}(M)=\operatorname{lip}_{0}(M)^{*} .
$$

Let us remark that $\operatorname{lip}_{0}(M)$ is usually not a unique predual: for instance, consider the well-known cases where $\mathcal{F}(M)=\ell_{1}$ (see e.g. [52, Example 3.10]), which has a plethora of non-isomorphic preduals.

The study of duality of Lipschitz-free spaces $\mathcal{F}(M)$ can be traced back to the 1960's and the first important result is due to Johnson [36], who proved that (4) holds for any compact $M$ when endowed with a Hölder metric. A powerful sufficient condition for (4) was identified much later by Weaver in [50], based on observations in $[5,32]$ : it is enough that $\operatorname{lip}_{0}(M)$ separates points of $M$ uniformly. It is then immediate from Goldstine's theorem that the separation constant is 1. Using this condition, Dalet proved (4) for compacts $M$ that are either countable [17] or ultrametric [18]. Weaver later gave a much simplified proof of Dalet's result for the countable case [52, Theorem 4.11]. In turn, three of the named authors used in [3, Theorem 4.3] an argument similar to Weaver's to show that (4) holds whenever $M$ is a compact 0-hyperbolic metric space whose length measure is 0 . The same proof can in fact be used, with only slight modifications, to show that (4) is true for any compact $M$ with $\mathcal{H}^{1}(M)=0$.

On the other hand, we may also identify necessary conditions for duality. By Lebesgue's fundamental theorem of calculus, there are no nontrivial locally flat functions on $[0,1]$, and therefore $\operatorname{lip}_{0}(M)$ is trivial whenever $M$ is geodesic. Godard provided a stronger necessary condition in [27] when he proved that $\mathcal{F}(M)$ is isomorphic to $L_{1}$ for any $M \subset \mathbb{R}$ with positive Lebesgue measure. Since biLipschitz equivalent metric spaces have isomorphic Lipschitz-free spaces, it follows that $\mathcal{F}(M)$ cannot be a dual space if $M$ contains a curve fragment - that is $M$ has 
to be purely 1-unrectifiable for $\mathcal{F}(M)$ to be a dual. More generally, this fact holds for all separable $M$.

Returning to the compact setting, Theorem 2.1 now allows us to fill that gap immediately as it shows that, when $M$ is compact, pure 1-unrectifiability of $M$ is also a sufficient condition for $\operatorname{lip}_{0}(M)$ to separate points of $M$ uniformly, and thus for (4) to hold. In fact, combining it with previously known implications, we deduce that this characterizes some well-known Banach space properties in Lipschitz-free spaces, like the Radon-Nikodým property or the Schur property.

It happens frequently in Lipschitz-free space theory that results for compact metric spaces can be extended to proper metric spaces, i.e. such that every closed ball is compact. This is also the case with the characterization of duality, but it requires a slight modification of the definition of the little Lipschitz space. For the remainder of this section, we will denote by $\operatorname{lip}_{0}(M)$ the space of all functions in $\operatorname{Lip}_{0}(M)$ that are locally flat and moreover flat at infinity, i.e. such that $\left\|f \uparrow_{M \backslash B_{r}(0)}\right\|_{L} \rightarrow 0$ as $r \rightarrow \infty$. Note that this condition is superfluous when $M$ is bounded.

Theorem 3.1. Let $M$ be a proper metric space. Then the following are equivalent:

(i) $M$ is purely 1-unrectifiable,

(ii) $\operatorname{lip}_{0}(M)$ separates points of $M$ uniformly,

(iii) $\operatorname{lip}_{0}(M)^{*}=\mathcal{F}(M)$,

(iv) $\mathcal{F}(M)$ is a dual space,

(v) $\mathcal{F}(M)$ has the Radon-Nikodym property,

(vi) $\mathcal{F}(M)$ has the Schur property,

(vii) $\mathcal{F}(M)$ contains no isomorphic copy of $L_{1}$.

Proof. (i) $\Rightarrow$ (ii): The compact case is given by Theorem 2.1. Let us now extend it to the proper case. Fix two points $p, q \in M$ and $\delta>0$. Let $r=d(p, q)$ and $K=B_{r+2 \delta}(p)$. Then $K$ is compact and purely 1-unrectifiable, so by Theorem 2.1 there exists $g \in B_{\operatorname{lip}(K)}$ such that $g(p)=0$ and $g(x) \geq d(p, x)-\delta$ for every $x \in K$. Now define $h: M \rightarrow \mathbb{R}$ by

$$
h(x)= \begin{cases}g(x) \wedge r & , \text { if } x \in K \\ r & , \text { if } x \notin K\end{cases}
$$

Note that $h(x)=r$ if $d(x, p) \geq r+\delta$, so it is easy to check that $h$ is 1-Lipschitz, locally flat, and flat at infinity. Clearly $h(q)-h(p) \geq r-\delta$ so letting $f=h-h(0)$ provides a map in $B_{\operatorname{lip}_{0}(M)}$ which separates $p$ and $q$ as required.

(ii) $\Rightarrow$ (iii): The proper case is proved in [18, Theorem 2.1].

(iii) $\Rightarrow$ (iv) is trivial.

(iv) $\Rightarrow(\mathrm{v}): \quad M$ is separable, hence so is $\mathcal{F}(M)$, and any separable dual Banach space has the RNP (see e.g. [47, Corollary 2.15]).

(iii) $\Rightarrow(v i)$ : This is implied by $\left[45\right.$, Proposition 8] (note that $\operatorname{lip}_{0}(M)$ in that result's statement refers to the space of uniformly locally flat functions, which includes our little Lipschitz space in both the compact and proper settings).

$(\mathrm{v}) \Rightarrow$ (vii) and (vi) $\Rightarrow$ (vii) follow from the fact that the Radon-Nikodým and the Schur properties are hereditary and preserved by isomorphisms, and $L_{1}$ fails both of them. $3.4]$.

(vii) $\Rightarrow(\mathrm{i})$ : As explained above, this follows from Godard's theorem [27, Corollary

The equivalent conditions in Theorem 3.1 imply a strong $\ell_{1}$-like behavior of $\mathcal{F}(M)$. In fact, a stronger quantitative version of the Schur property called the 1-strong Schur property is fulfilled in this case (see [45] for the definition and Proposition 17 therein for the immediate proof). Let us point out that $\mathcal{F}(M)$ is also $w^{*}$ 
asymptotically uniformly convex with power type 1 modulus in this case (see e.g. [46, Proposition 4.4.2]).

There are several other Banach space properties, like the Krein-Milman property, that lie between the RNP or the Schur property and the non-containment of $L_{1}$ and are therefore equivalent to them under the hypothesis of Theorem 3.1. We will see later that this equivalence holds in a more general case, so we withhold the discussion of these properties until Section 4.

3.1. Preduals of $\mathcal{F}(M)$ with additional structure. For proper $M$, Theorem 3.1 shows that $\operatorname{lip}_{0}(M)$ is a predual of $\mathcal{F}(M)$ whenever there is one. This doesn't preclude the possibility of there being other preduals. In fact, $\operatorname{lip}_{0}(M)$ is never a unique predual unless it is finite-dimensional. Indeed, $\operatorname{lip}_{0}(M)$ embeds almostisometrically into $c_{0}$ by [18, Lemma 3.9], which implies that it is an M-embedded Banach space (see [33, Section III.1] for the notion and the proof of that fact). Thus [33, Proposition III.2.10] proves that $\operatorname{lip}_{0}(M)$ is not a unique predual. Moreover, $\mathcal{F}(M)=\operatorname{lip}_{0}(M)^{*}$ is then L-embedded and so [33, Proposition IV.1.9] shows that $\operatorname{lip}_{0}(M)$ is the unique predual of $\mathcal{F}(M)$ that is M-embedded.

We shall now prove that $\operatorname{lip}_{0}(M)$ is also the unique predual that satisfies the constraint of having a lattice structure. Recall that the spaces $\operatorname{Lip}_{0}(M)$ and $\operatorname{Lip}(M)$ are vector lattices under the operations $\vee$ and $\wedge$ of pointwise maximum and minimum, with $\operatorname{Lip}(M)=\operatorname{Lip}_{0}(M)+\operatorname{span}\left\{1_{M}\right\}$ where $1_{M}$ denotes the function on $M$ that takes the constant value 1 . A similar statement holds for $\operatorname{lip}_{0}(M)$ and $\operatorname{lip}(M)$.

We will say that $Y$ is a linear sublattice of $\operatorname{Lip}_{0}(M)$ or $\operatorname{Lip}(M)$ if it is a linear subspace such that $f \vee g \in Y$ (and thus also $f \wedge g \in Y$ ) whenever $f, g \in Y$. Following [52], we say that a linear sublattice of $\operatorname{Lip}_{0}(M)$ is shiftable if $f \vee\left(g-c \cdot 1_{M}\right) \in Y$ whenever $f, g \in Y$ and $c \geq 0$. The artificial-looking condition $c \geq 0$ ensures that the resulting function still vanishes at 0 . It is straightforward to check that $Y$ is a shiftable linear sublattice of $\operatorname{Lip}_{0}(M)$ if and only if $Y+\operatorname{span}\left\{1_{M}\right\}$ is a linear sublattice of $\operatorname{Lip}(M)$. Thus, shiftable linear sublattices are invariant with respect to a change of base point in $M$. This is not true for all sublattices: consider e.g. the one-dimensional space generated by the function $x \mapsto d(x, 0)$.

Let us say that a real-valued function $f$ defined on a metric space is coercive if $|f(x)| \rightarrow \infty$ as $d(x, 0) \rightarrow \infty$. Notice that coercivity does not depend on the choice of base point, and that any function defined on a bounded metric space is coercive by vacuity. Thus, we will only need the next lemma for the unbounded case:

Lemma 3.2. Let $M$ be a proper metric space and let $Y$ be a closed, $w^{*}$-dense linear sublattice of $\operatorname{Lip}_{0}(M)$. Then $Y$ contains a positive coercive function.

Proof. Let us first verify the following simple claim:

Claim. Given a positive $f \in B_{\operatorname{Lip}_{0}(M)}, r>0$ and $\varepsilon>0$, there is a positive $g \in B_{Y}$ such that $|f(x)-g(x)| \leq \varepsilon$ for every $x \in B_{r}(0)$.

Indeed, we can find a finite $\varepsilon / 3$-net $A$ in $B_{r}(0)$ because it is a compact set. Since $B_{Y}$ is $w^{*}$-dense in $B_{\operatorname{Lip}_{0}(M)}$, there is $h \in B_{Y}$ such that $|f(y)-h(y)| \leq \varepsilon / 3$ for every $y \in A$. Now if $x \in B_{r}(0)$, find $y \in A$ with $d(x, y) \leq \varepsilon / 3$ and we have

$$
|f(x)-h(x)| \leq|f(x)-f(y)|+|f(y)-h(y)|+|h(y)-h(x)| \leq \varepsilon .
$$

Then $g=h \vee 0$ satisfies the requirements.

Now use the Claim to obtain positive functions $f_{n} \in B_{Y}$ for every $n \in \mathbb{N}$ such that $f_{n}(x) \geq d(x, 0)-1$ for every $x \in B_{4^{n}}(0)$, and let $f=\sum_{n=1}^{\infty} f_{n} / 2^{n}$. Then $f \in Y$ because $Y$ is closed, and if $x \in M$ is such that $d(x, 0)>4^{k}$ for some $k \in \mathbb{N}$ then

$$
f(x) \geq \sum_{n=k+1}^{\infty} \frac{f_{n}(x)}{2^{n}} \geq \sum_{n=k+1}^{\infty} \frac{d(x, 0)-1}{2^{n}}>\frac{4^{k}-1}{2^{k}}>2^{k}-1 .
$$


This shows that $f$ is coercive.

For the next result we need to introduce some notation. Let

$$
\widetilde{M}=\{(x, y) \in M \times M: x \neq y\}
$$

be the set of pairs of different points of $M$, with the topology inherited from $M \times M$. For $(x, y) \in \widetilde{M}$ denote

$$
m_{x y}=\frac{\delta(x)-\delta(y)}{d(x, y)}
$$

which is a norm 1 element of $\mathcal{F}(M)$, usually called the elementary molecule determined by $x$ and $y$. The de Leeuw map is the mapping $\Phi$ that takes a function $f \in \operatorname{Lip}_{0}(M)$ to the function $\Phi f: \widetilde{M} \rightarrow \mathbb{R}$ defined by

$$
\Phi f(x, y)=\left\langle m_{x y}, f\right\rangle=\frac{f(x)-f(y)}{d(x, y)} .
$$

Clearly $\Phi f$ is continuous and bounded, with $\|\Phi f\|_{\infty}=\|f\|_{L}$, and so it can be identified with its continuous extension to $\beta \widetilde{M}$, the Stone-Čech compactification of $\widetilde{M}$. Thus we may regard $\Phi$ as a linear isometry from $\operatorname{Lip}_{0}(M)$ into $C(\beta \widetilde{M})$.

With the required notation in place, we may state and prove the following variation of [52, Theorem 3.43]:

Lemma 3.3. Let $M$ be a proper metric space and let $Y$ be a shiftable linear sublattice of $\operatorname{Lip}_{0}(M)$ that contains a coercive function. Let $\zeta \in \beta \widetilde{M}$ and assume that there exists $g \in Y$ such that $\Phi g(\zeta) \neq 0$. Then $\zeta \in \widetilde{M}$ if and only if $\Phi f_{i}(\zeta) \rightarrow \Phi f(\zeta)$ for every bounded net $\left(f_{i}\right)$ in $Y$ that converges pointwise to $f \in Y$.

Proof. The forward implication is obvious, since $\zeta=(x, y) \in \widetilde{M}$ implies $\Phi f_{i}(\zeta)=$ $\left\langle m_{x y}, f_{i}\right\rangle \rightarrow\left\langle m_{x y}, f\right\rangle=\Phi f(\zeta)$ whenever $f_{i} \stackrel{w^{*}}{\longrightarrow} f$ in $\operatorname{Lip}_{0}(M)$. For the backward implication, choose any $\zeta \in \beta \widetilde{M} \backslash \widetilde{M}$ and we will show that there is a bounded sequence $\left(f_{n}\right)$ in $Y$ that converges pointwise to $f$ but fails $\Phi f_{n}(\zeta) \rightarrow \Phi f(\zeta)$. We will follow the proof of [52, Theorem 3.43] closely.

Let $\left(x_{i}, y_{i}\right)$ be a net in $\widetilde{M}$ that converges to $\zeta$; we may assume that $x_{i}$ and $y_{i}$ converge to elements $\xi$ and $\eta$ of $\beta M$. We may also take $g \geq 0$ : since $Y$ is a sublattice, $g^{+}=g \vee 0$ and $g^{-}=(-g) \vee 0$ belong to $Y$ and are non-negative, and at least one of $\Phi g^{+}(\zeta), \Phi g^{-}(\zeta)$ must be different from 0 . Write $d(\zeta)=\lim _{i} d\left(x_{i}, y_{i}\right) \in[0, \infty]$, and notice that $|g(\xi)-g(\eta)|=|\Phi g(\zeta)| \cdot d(\zeta)$. We now distinguish three cases:

Case 1: $d(\zeta)=0$. Then $g(\xi)=g(\eta)$; denote this value by $a$. Suppose first that $a<\infty$, let

$$
g_{n}=\left(g \wedge\left(1+\frac{1}{n}\right) a \cdot 1_{M}\right) \vee\left(1-\frac{1}{n}\right) a \cdot 1_{M}
$$

and $f_{n}=g_{n}-g_{n}(0) \cdot 1_{M} \in Y$. Then $\left\|f_{n}\right\|_{L} \leq\|g\|_{L}$ and $f_{n}(x)$ decreases to 0 for every $x \in M$. However, for each $n$ we have $\left|g\left(x_{i}\right)-a\right|<\frac{1}{n}$ and $\left|g\left(y_{i}\right)-a\right|<\frac{1}{n}$ eventually, so $\Phi f_{n}\left(x_{i}, y_{i}\right)=\Phi g_{n}\left(x_{i}, y_{i}\right)=\Phi g\left(x_{i}, y_{i}\right)$ eventually and $\Phi f_{n}(\zeta)=\Phi g(\zeta)$. That is, $f_{n} \rightarrow 0$ pointwise but $\Phi f_{n}(\zeta) \rightarrow \Phi g(\zeta) \neq 0$.

If $a=\infty$, take $f_{n}=\left(g-n \cdot 1_{M}\right) \vee 0 \in Y$ instead. Again, $\left\|f_{n}\right\|_{L} \leq\|g\|_{L}$ and $f_{n} \rightarrow 0$ pointwise, yet $g\left(x_{i}\right), g\left(y_{i}\right)>n$ eventually and so $\Phi f_{n}(\zeta)=\Phi g(\zeta)$ for each $n$.

Case 2: $d(\zeta)=\infty$. Take $f_{n}=\left(g-n \cdot 1_{M}\right) \vee 0 \in Y$ again. In this case,

$$
\lim _{i}\left|\Phi f_{n}\left(x_{i}, y_{i}\right)-\Phi g\left(x_{i}, y_{i}\right)\right| \leq \lim _{i} \frac{2 n}{d\left(x_{i}, y_{i}\right)}=0
$$

since $\left\|f_{n}-g\right\|_{\infty} \leq n$, and so $\Phi f_{n}(\zeta)=\Phi g(\zeta)$ for fixed $n$. 
Case 3: $0<d(\zeta)<\infty$. We will show that we may assume that $g(\xi)=g(\eta)=\infty$, so we may then take $f_{n}=\left(g-n \cdot 1_{M}\right) \vee 0$ yet again and apply the argument from Case 1.

Indeed, assume otherwise. Then $g(\xi)$ and $g(\eta)$ are both finite since $|g(\xi)-g(\eta)|=$ $|\Phi g(\zeta)| \cdot d(\zeta)<\infty$. Now, observe that $\xi$ and $\eta$ cannot both belong to $M$ as $\zeta \notin \widetilde{M}$. If, say, $\xi \notin M$, then $d(\xi, 0)=\infty$ because $M$ is proper (if $d(\xi, 0)<r$, then the compactness of $B_{r}(0)$ would imply $\left.\xi \in M\right)$, and $d(\zeta)<\infty$ implies that $d(\eta, 0)=\infty$ as well. By hypothesis, there is a coercive function $v \in Y$, and we may assume $v \geq 0$ by replacing it with $|v| \in Y$. Thus $v(\xi)=v(\eta)=\infty$. If $\Phi v(\zeta) \neq 0$, then take $v$ instead of $g$ to get the desired contradiction. Otherwise, use the function $g+v$.

We may now prove the desired uniqueness result.

Theorem 3.4. Let $M$ be a proper metric space. If $Y$ is a closed shiftable linear sublattice of $\operatorname{Lip}_{0}(M)$ such that $Y^{*}=\mathcal{F}(M)$, then $Y=\operatorname{lip}_{0}(M)$.

Proof. We consider the weak* topology in $\mathcal{F}(M)$ induced by $Y$. Let $\zeta \in \beta \widetilde{M}$, and let $\left(x_{i}, y_{i}\right)$ be a net in $\widetilde{M}$ that converges to $\zeta$. Then $\left(m_{x_{i} y_{i}}\right)$ is a net in the unit ball of $\mathcal{F}(M)$, which is a $w^{*}$-compact set, so we can replace $\left(m_{x_{i} y_{i}}\right)$ with a subnet such that $m_{x_{i} y_{i}} \stackrel{w^{*}}{\longrightarrow} \mu$ for some $\mu \in \mathcal{F}(M)$. This means that

$$
\langle\mu, f\rangle=\lim _{i}\left\langle m_{x_{i} y_{i}}, f\right\rangle=\lim _{i} \Phi f\left(x_{i}, y_{i}\right)=\Phi f(\zeta)
$$

for every $f \in Y$. Now let $\left(f_{i}\right)$ be a bounded net in $Y$ that converges pointwise to $f \in Y$. Then $f_{i} \stackrel{w^{*}}{\longrightarrow} f$ in the weak* topology of $\operatorname{Lip}_{0}(M)$, and so $\left\langle\mu, f_{i}\right\rangle \rightarrow\langle\mu, f\rangle$, that is, $\Phi f_{i}(\zeta) \rightarrow \Phi f(\zeta)$.

We have thus shown that $Y$ satisfies the condition in Lemma 3.3. Since it also contains a coercive function by Lemma 3.2, it follows that either $\zeta \in \widetilde{M}$ or $\left.\mu\right|_{Y}=0$. Therefore $\Phi f(\zeta)=0$ for every $f \in Y$ and every $\zeta \in \beta \widetilde{M} \backslash \widetilde{M}$. By [52, Proposition 4.20], this is equivalent to $Y \subset \operatorname{lip}_{0}(M)$. But $\operatorname{lip}_{0}(M)$ is also a predual of $\mathcal{F}(M)$ by Theorem 3.1, so $Y=\operatorname{lip}_{0}(M)$.

\section{The RAdON-NIKODÝM PROPERTY IN LIPSCHITZ-FREE SPACES}

Very recently, a method of "compact reduction" was introduced in $[2,26]$ to prove some properties of Lipschitz-free spaces by considering only the compact case. Let us say informally that a Banach space property $(\mathrm{P})$ is compactly determined in Lipschitz-free spaces if for every metric space $M$, the free space $\mathcal{F}(M)$ has $(\mathrm{P})$ whenever $\mathcal{F}(K)$ has $(\mathrm{P})$ for every compact subset $K \subset M$. For instance, it is proved in [2, Corollary 2.6] that the Schur property satisfies such a statement (see also [2, Corollary 2.9] for $\ell_{1}$-saturation).

In order to extend the equivalences in Theorem 3.1 to the noncompact case, it would be enough to show that the corresponding properties are compactly determined. Unfortunately, the property " $\mathcal{F}(M)$ is (isometrically) a dual space" is not determined by compact subsets of $M$. There are indeed examples of uniformly discrete metric spaces $M$ for which $\mathcal{F}(M)$ is not isometric to any dual Banach space (see [24, Example 5.8] and [11, Remark 6.4]), although any compact subset $K \subset M$ is finite so that $\mathcal{F}(K)$ is finite-dimensional. However, we will prove in this section that the RNP is compactly determined in Lipschitz-free spaces, and its equivalence with the Schur property will follow by compact reduction.

4.1. Compact determination of the RNP. The Radon-Nikodým property admits many equivalent formulations; see e.g. [20, Section VII.6]. Our arguments in 
this section will be based on its characterization in terms of martingales in Bochner spaces. Let us recall the notions involved in it.

Let $(\Omega, \mathcal{A}, \mathbb{P})$ be a probability space and let $X$ be a Banach space. We denote by $L_{1}(\Omega, \mathcal{A}, \mathbb{P} ; X)$ the space of (equivalence classes of) Bochner measurable functions $f: \Omega \rightarrow X$ such that $\int_{\Omega}\|f\|_{X} d \mathbb{P}<\infty$ equipped as usual with the norm

$$
\|f\|_{L_{1}(\Omega, \mathcal{A}, \mathbb{P} ; X)}=\mathbb{E}\left[\|f\|_{X}\right]=\int_{\Omega}\|f\|_{X} d \mathbb{P} .
$$

In the sequel, we will suppress notation and write simply $L_{1}(X)$, or $L_{1}(\mathcal{A} ; X)$ when the $\sigma$-algebra $\mathcal{A}$ needs to be emphasized.

We recall that a sequence $\left(M_{n}\right)_{n=0}^{\infty}$ in $L_{1}(\mathcal{A} ; X)$ is called a martingale if there exists an increasing sequence $\left(\mathcal{A}_{n}\right)_{n=0}^{\infty}$ of $\sigma$-subalgebras of $\mathcal{A}$ (called a filtration) such that for each $n \geq 0, M_{n}$ is $\mathcal{A}_{n}$-measurable and satisfies

$$
M_{n}=\mathbb{E}^{\mathcal{A}_{n}}\left(M_{n+1}\right),
$$

where $\mathbb{E}^{\mathcal{A}_{n}}$ denotes the $X$-valued conditional expectation (see e.g. [47, Section 1.2]). We say moreover that $\left(M_{n}\right)_{n=0}^{\infty}$ is uniformly integrable if the sequence of nonnegative random variables $\left(\left\|M_{n}\right\|_{X}\right)_{n=0}^{\infty}$ is uniformly integrable. More precisely, this means that $\left(\left\|M_{n}\right\|_{X}\right)_{n=0}^{\infty}$ is bounded in $L_{1}(\mathbb{R})$ and that, for any $\varepsilon>0$, there is a $\delta>0$ such that

$$
\forall A \in \mathcal{A}, \quad \mathbb{P}(A)<\delta \Longrightarrow \sup _{n \geq 0} \int_{A}\left\|M_{n}\right\|_{X} d \mathbb{P}<\varepsilon .
$$

Whenever $T: X \rightarrow Y$ is a bounded linear operator, we get a well-defined bounded linear operator $\widetilde{T}: L_{1}(X) \rightarrow L_{1}(Y)$ defined by $\widetilde{T}(f)=T \circ f$ with $\|\widetilde{T}\|=$ $\|T\|$. An elementary but important fact is that $\widetilde{T}$ commutes with any conditional expectation operator $\mathbb{E}^{\mathcal{A}_{n}}$. In the sequel, we will abuse notation and denote $\widetilde{T}$ by $T$.

A Banach space $X$ has the RNP if and only if every uniformly integrable $X$ valued martingale converges in $L_{1}(X)$ (see [47, Theorem 2.9]). We will use this characterization in order to prove that the RNP is compactly determined in Lipschitzfree spaces. Our way to the proof will be similar to the method used in [2].

For the remainder of this section, fix a complete metric space $(M, d)$ and a probability space $(\Omega, \mathcal{A}, \mathbb{P})$.

Definition 4.1. Inspired by the terminology from [2], we say that a collection $W \subset L_{1}(\mathcal{F}(M))$ of random variables $F:(\Omega, \mathcal{A}, \mathbb{P}) \rightarrow \mathcal{F}(M)$ has the mean Kalton property if for every $\varepsilon, r>0$, there exists a finite set $E \subset M$ such that

$$
d\left(F, L_{1}\left(\mathcal{F}\left([E]_{r}\right)\right)\right) \leq \varepsilon \quad \text { for all } F \in W,
$$

and we say that $W$ is mean-tight if, for every $\varepsilon>0$, there exist a compact $K \subset M$ such that

$$
d\left(F, L_{1}(\mathcal{F}(K))\right) \leq \varepsilon \quad \text { for all } F \in W .
$$

It is clear that if $W$ is mean-tight then it has the mean Kalton property. We will now show that the converse is actually also true.

Proposition 4.2. Let $W \subset L_{1}(\mathcal{F}(M))$ be a collection of random variables with the mean Kalton property. Then $W$ is mean-tight. More precisely, for every $\varepsilon>0$ there exist a compact $K \subset M$ and a mapping $T: W \rightarrow L_{1}(\mathcal{F}(K))$ such that

- $\mathbb{E}(\|F-T(F)\|) \leq \varepsilon$ for every $F \in W$, and

- there is a sequence of bounded linear operators $T_{n}: \mathcal{F}(M) \rightarrow \mathcal{F}(M), n \in \mathbb{N}$ such that

$$
\lim _{n \rightarrow \infty} \sup _{F \in W} \mathbb{E}\left(\left\|T_{n}(F)-T(F)\right\|\right)=0
$$


Proof. We follow the proof of [2, Theorem 3.2] closely. Suppose first that $M$ is bounded, let $R=\operatorname{diam}(M)$ and fix $\varepsilon \in\left(0, \frac{1}{3}\right)$. Set $\varepsilon_{0}=\varepsilon$ and $\delta_{0}=R$, and for $n \geq 1$ let $\varepsilon_{n}=2^{-n} \varepsilon$ and $\delta_{n}=R \cdot\left(\frac{1}{\varepsilon_{n}}-2\right)^{-1}$. Let also $K_{0}=M$ and $S_{0}$ be the identity operator on $\mathcal{F}(M)$. We will now construct, for $n \geq 1$, finite subsets $E_{n} \subset M$ containing 0 , closed subsets $K_{n} \subset M$, and operators $S_{n}: \mathcal{F}(M) \rightarrow \mathcal{F}(M)$ such that

(a) $K_{n}=K_{n-1} \cap\left[E_{n}\right]_{2 \delta_{n}}$,

(b) the operators $S_{n}$ commute with each other,

(c) $T_{n}(\mathcal{F}(M)) \subset \mathcal{F}\left(K_{n}\right)$, and

(d) $\mathbb{E}\left(\left\|T_{n-1}(F)-T_{n}(F)\right\|\right) \leq \varepsilon_{n}$ for all $F \in W$, where $T_{n}=S_{n} \circ S_{n-1} \circ \ldots \circ S_{0}$.

We proceed by induction. Let $n \geq 1$ and suppose that $E_{n-1}$ and $S_{n-1}$ have already been constructed. By the mean Kalton property, we may find a finite set $E_{n} \subset M$ such that $0 \in E_{n}$ and

$$
\left\|T_{n-1}\right\| \cdot \sup _{F \in W} d\left(F, L_{1}\left(\mathcal{F}\left(\left[E_{n}\right]_{\delta_{n}}\right)\right)\right)<\varepsilon_{n}^{2} .
$$

Let $K_{n}$ be as in (a). Now consider the function $h_{n}: M \rightarrow \mathbb{R}$ given by

$$
h_{n}(x)=0 \vee\left(1-\delta_{n}^{-1} \cdot d\left(x,\left[E_{n}\right]_{\delta_{n}}\right)\right)
$$

for $x \in M$, and define the operator $S_{n}$ by

$$
\left\langle S_{n} \mu, f\right\rangle=\left\langle\mu, f \cdot h_{n}\right\rangle
$$

for $\mu \in \mathcal{F}(M)$ and $f \in \operatorname{Lip}_{0}(M)$. Clearly $0 \leq h_{n} \leq 1,\left\|h_{n}\right\|_{L} \leq \delta_{n}^{-1}$ and $\operatorname{supp}\left(h_{n}\right) \subset$ $\left[E_{n}\right]_{2 \delta_{n}}$, so by [4, Proposition 2.4] we have $\left\|S_{n}\right\| \leq 1+R / \delta_{n}=\varepsilon_{n}^{-1}-1$. It is also clear that the restriction of $S_{n}$ to $\mathcal{F}\left(\left[E_{n}\right]_{\delta_{n}}\right)$ is the identity, and that (b) holds for all $S_{n}$ defined in this way. Moreover, by formula (3) in [4] and inductive hypothesis (c) we have

$$
T_{n}(\mathcal{F}(M))=S_{n}\left(T_{n-1}(\mathcal{F}(M))\right) \subset S_{n}\left(\mathcal{F}\left(K_{n-1}\right)\right) \subset \mathcal{F}\left(K_{n-1} \cap\left[E_{n}\right]_{2 \delta_{n}}\right)=\mathcal{F}\left(K_{n}\right)
$$

so (c) holds. Finally let us check (d). Fix $F \in W$, then by (5) there is $G \in$ $L_{1}\left(\mathcal{F}\left(\left[E_{n}\right]_{\delta_{n}}\right)\right)$ such that $\mathbb{E}\left(\left\|T_{n-1}(F)-T_{n-1}(G)\right\|\right)<\varepsilon_{n}^{2}$, and we have $T_{n}(G)=$ $T_{n-1}\left(S_{n}(G)\right)=T_{n-1}(G)$ by (b). So

$$
\begin{aligned}
\mathbb{E}\left(\left\|T_{n-1}(F)-T_{n}(F)\right\|\right) & \leq \mathbb{E}\left(\left\|T_{n-1}(F)-T_{n-1}(G)\right\|\right)+\mathbb{E}\left(\left\|T_{n}(G)-T_{n}(F)\right\|\right) \\
& \leq\left(1+\left\|S_{n}\right\|\right) \mathbb{E}\left(\left\|T_{n-1}(F)-T_{n-1}(G)\right\|\right) \leq \varepsilon_{n} .
\end{aligned}
$$

The construction is thus complete.

Now let $K=\bigcap_{n=1}^{\infty} K_{n}$. Since $\delta_{n} \rightarrow 0$, (a) implies that $K$ is totally bounded. Then, since $K$ is closed and $M$ is complete, $K$ is compact. For every $F \in W$, it follows from $(\mathrm{d})$ that the sequence $\left(T_{n}(F)\right)$ is Cauchy so it converges to some element of $L_{1}(\mathcal{F}(M))$. Denote the limit by $T(F)$. This gives us a map

$$
T: W \longrightarrow \bigcap_{n=1}^{\infty} L_{1}\left(\mathcal{F}\left(K_{n}\right)\right)=L_{1}(\mathcal{F}(K)),
$$

where the equality holds by Pettis' measurability theorem (see [20, p. 42]) and the fact that $\bigcap_{n} \mathcal{F}\left(K_{n}\right)=\mathcal{F}(K)$ (see [4, Theorem 2.1]). Finally, notice that

$$
\mathbb{E}\left(\left\|F-T_{n}(F)\right\|\right) \leq \sum_{i=1}^{n} \mathbb{E}\left(\left\|T_{i-1}(F)-T_{i}(F)\right\|\right)<\varepsilon
$$

by $(\mathrm{d})$, and therefore $\mathbb{E}(\|F-T(F)\|) \leq \varepsilon$. Similarly $\mathbb{E}\left(\left\|T_{n}(F)-T(F)\right\|\right) \leq \varepsilon_{n}$ for every $n$. This completes the proof of the bounded case.

If $M$ is unbounded, then we simply replace the initial construction step as follows. By assumption we can find a finite set $E_{0} \subset M$ such that $d\left(F, L_{1}\left(\mathcal{F}\left(\left[E_{0}\right]_{1}\right)\right)\right)<\frac{\varepsilon}{4}$ 
for every $F \in W$. Set $R=2(\operatorname{diam}(E)+1)$ and $K_{0}=B_{R}(0)$, consider the function $h_{0}: M \rightarrow \mathbb{R}$ given by

$$
h_{0}(x)=0 \vee\left(1 \wedge\left(2-2 R^{-1} d(x, 0)\right)\right)
$$

for $x \in M$, and let $S_{0}: \mathcal{F}(M) \rightarrow \mathcal{F}\left(K_{0}\right)$ be defined by $\left\langle S_{0} \mu, f\right\rangle=\left\langle\mu, f \cdot h_{0}\right\rangle$ for $f \in \operatorname{Lip}_{0}(M)$. Then, similarly as above, we have $\left\|h_{0}\right\|_{L} \leq 2 R^{-1},\left\|S_{0}\right\| \leq 3$, and thus

$$
\mathbb{E}\left(\left\|F-S_{0}(F)\right\|\right) \leq\left(1+\left\|S_{0}\right\|\right) \frac{\varepsilon}{4}<\varepsilon
$$

for every $F \in W$. We can then continue the inductive construction as above.

Next, we obtain a probabilistic version of Kalton's lemma [38, Lemma 4.5] for martingales in place of weakly null sequences. This is the main new technical tool needed to prove compact determination of the RNP.

Proposition 4.3. Suppose that $M$ is bounded. Then every $L_{1}(\mathcal{F}(M))$-bounded martingale has the mean Kalton property.

Proof. Assume towards a contradiction that there exist $r>0, \varepsilon>0$, and an $L_{1}(\mathcal{F}(M))$-bounded martingale $\left(\tilde{M}_{n}\right)_{n=0}^{\infty}$ adapted to a filtration $\left(\tilde{\mathcal{A}}_{n}\right)_{n=0}^{\infty}$ such that for every finite subset $E \subset M$, there exists $n \in \mathbb{N}$ with

$$
d\left(\tilde{M}_{n}, L_{1}\left(\mathcal{F}\left([E]_{r}\right)\right)\right)=d\left(\tilde{M}_{n}, L_{1}\left(\tilde{\mathcal{A}}_{n} ; \mathcal{F}\left([E]_{r}\right)\right)\right)>4 \varepsilon .
$$

First we use a standard approximation technique to replace $\left(\tilde{M}_{n}\right)_{n=0}^{\infty}$ with a martingale $\left(M_{n}\right)_{n=0}^{\infty}$ adapted to a filtration $\left(\mathcal{A}_{n}\right)_{n=0}^{\infty}$ where each $\mathcal{A}_{n}$ is finite. Here is the technique: for each $n \geq 0$, choose an $\tilde{\mathcal{A}}_{n}$-measurable simple function $G_{n}$ such that $\left\|\tilde{M}_{n}-G_{n}\right\|_{L_{1}}<\varepsilon$. Let $\mathcal{A}_{n}$ be the (finite) $\sigma$-algebra generated by $G_{0}, G_{1}, \ldots, G_{n}$. Then, using $\mathcal{A}_{n} \subset \tilde{\mathcal{A}}_{n}, \mathcal{A}_{n+1}$ and the tower property of conditional expectations, we can see that $\left(M_{n}\right)_{n=0}^{\infty}:=\left(\mathbb{E}^{\mathcal{A}_{n}}\left(\tilde{M}_{n}\right)\right)_{n=0}^{\infty}$ is an $L_{1}(\mathcal{F}(M))$-bounded martingale adapted to $\left(\mathcal{A}_{n}\right)_{n=0}^{\infty}$. Now observe that $\left\|\tilde{M}_{n}-M_{n}\right\|_{L_{1}}<2 \varepsilon$, which implies, for every finite $E \subset M$, there exists $n \in \mathbb{N}$ with

$$
d\left(M_{n}, L_{1}\left(\mathcal{F}\left([E]_{r}\right)\right)\right)=d\left(M_{n}, L_{1}\left(\mathcal{A}_{n} ; \mathcal{F}\left([E]_{r}\right)\right)\right)>2 \varepsilon .
$$

By using density of the finitely supported elements in $\mathcal{F}(M)$, we may replace $\left(M_{n}\right)_{n}$ with a stochastic process $\left(\bar{M}_{n}\right)_{n}$ adapted to $\left(\mathcal{A}_{n}\right)_{n=0}^{\infty}$ satisfying

- $\bar{M}_{n}$ is constant on each atom of $\mathcal{A}_{n}$ and not just essentially constant,

- $\bar{M}_{n}(\omega)$ is finitely supported for every $n \in \mathbb{N}$ and $\omega \in \Omega$,

- $\left(\bar{M}_{n}\right)_{n}$ is $L_{1}(\mathcal{F}(M))$-bounded,

- for every finite $E \subset M$, there exists $n \in \mathbb{N}$ with

$$
d\left(\bar{M}_{n}, L_{1}\left(\mathcal{A}_{n} ; \mathcal{F}\left([E]_{r}\right)\right)\right)>\varepsilon
$$

- for every $n \leq i,\left\|\mathbb{E}^{\mathcal{A}_{n}}\left(\bar{M}_{i}\right)-\bar{M}_{n}\right\|_{L_{1}} \leq 2^{-n} \wedge \varepsilon$.

Notice that the last condition implies that $\left(\overline{M_{n}}\right)_{n=0}^{\infty}$ is a quasi-martingale; see [47, Remark 2.16] for more details.

Set $N_{0}:=0$ and $E_{0}:=\bigcup_{n \leq N_{0}} \bigcup_{\omega \in \Omega} \operatorname{supp}\left(\bar{M}_{n}(\omega)\right)$. It is clear that $E_{0}$ is finite. Then there exists $N_{1}>N_{0}$ such that $d\left(\bar{M}_{N_{1}}, L_{1}\left(\mathcal{A}_{N_{1}} ; \mathcal{F}\left(\left[E_{0}\right]_{r}\right)\right)\right)>\varepsilon$. We let $E_{1}:=\bigcup_{n \leq N_{1}} \bigcup_{\omega \in \Omega} \operatorname{supp}\left(\bar{M}_{n}(\omega)\right)$, which is finite as well. Then there exists $N_{2}>$ $N_{1}$ such that $d\left(\bar{M}_{N_{2}}, L_{1}\left(\mathcal{A}_{N_{2}} ; \mathcal{F}\left(\left[E_{1}\right]_{r}\right)\right)\right)>\varepsilon$. Continuing in this way, we will get an increasing sequence of integers $\left(N_{i}\right)_{i=1}^{\infty} \subset \mathbb{N}$ and a sequence of finite sets $E_{0} \subset E_{1} \subset E_{2} \subset \cdots \subset M$ such that, setting $M_{i}^{\prime}:=\bar{M}_{N_{i}}$,

- $\operatorname{supp}\left(M_{i}^{\prime}(\omega)\right) \subset E_{i}$ for every $\omega \in \Omega$,

- $\left(M_{i}^{\prime}\right)_{i}$ is $L_{1}(\mathcal{F}(M))$-bounded,

- $d\left(M_{i+1}^{\prime}, L_{1}\left(\mathcal{A}_{N_{i+1}} ; \mathcal{F}\left(\left[E_{i}\right]_{r}\right)\right)\right)>\varepsilon$, 
- for all $n \leq i,\left\|\mathbb{E}^{\mathcal{A}_{N_{n}}}\left(M_{i}^{\prime}\right)-M_{n}^{\prime}\right\|_{L_{1}} \leq 2^{-N_{n}} \leq 2^{-n}$.

By the Hahn-Banach theorem, for each $i \geq 1$ there is $\tilde{f}_{i} \in L_{\infty}\left(\mathcal{A}_{N_{i}} ; \operatorname{Lip}_{0}(M)\right)=$ $L_{1}\left(\mathcal{A}_{N_{i}} ; \mathcal{F}(M)\right)^{*}$ such that

- $\left\|\tilde{f}_{i}\right\|_{L_{\infty}\left(\operatorname{Lip}_{0}(M)\right)} \leq 1$,

- $\tilde{f}_{i}(\omega)$ vanishes on $\left[E_{i-1}\right]_{r}$ for every $\omega \in \Omega$,

- $\mathbb{E}\left(\left\langle\tilde{f}_{i}, M_{i}^{\prime}\right\rangle\right)>\varepsilon$.

For each $\omega \in \Omega$, using the McShane-Whitney extension theorem (see [52, Theorem 1.33] for instance) we obtain a Lipschitz map $f_{i}(\omega): M \rightarrow \mathbb{R}$ so that

- $f_{i}(\omega)$ vanishes on $\left[E_{i-1}\right]_{r}$,

- $f_{i}(\omega)$ agrees with $\tilde{f}_{i}(\omega)$ on $E_{i}$,

- $\operatorname{supp}\left(f_{i}(\omega)\right) \subset\left[E_{i}\right]_{r}$,

- $\left\|f_{i}(\omega)\right\|_{L} \leq C\left\|\tilde{f}_{i}(\omega)\right\|_{L} \leq C$,

where $C=1 \vee(\operatorname{rad}(M) / r)$. Moreover, since $\mathcal{A}_{N_{i}}$ is finite, the above can be done so that $f_{i}$ is $\mathcal{A}_{N_{i}}$-measurable. Notice that the functions $\left(f_{i}(\omega)\right)_{i}$ have pairwise disjoint supports, therefore

$$
\left\|\sum_{n=1}^{i} f_{n}(\omega)\right\|_{L}=\left\|\sum_{n=1}^{i} f_{n}^{+}(\omega)-\sum_{n=1}^{i} f_{n}^{-}(\omega)\right\|_{L}=\left\|\bigvee_{n=1}^{i} f_{n}^{+}(\omega)-\bigvee_{n=1}^{i} f_{n}^{-}(\omega)\right\|_{L} \leq 2 C .
$$

Here we denote $f_{n}^{+}(\omega)=0 \vee f_{n}(\omega)$ and $f_{n}^{-}(\omega)=0 \vee\left(-f_{n}(\omega)\right)$ pointwise. Thus, we have for every $i \geq 1$

$$
\begin{aligned}
2 C\left\|M_{i}^{\prime}\right\|_{L_{1}} & \geq \mathbb{E}\left(\left\langle\sum_{n=1}^{i} f_{n}, M_{i}^{\prime}\right\rangle\right) \\
& =\mathbb{E}\left(\sum_{n=1}^{i}\left\langle f_{n}, \mathbb{E}^{\mathcal{A}_{N_{n}}}\left[M_{i}^{\prime}\right]\right\rangle\right) \\
& \geq \mathbb{E}\left(\sum_{n=1}^{i}\left\langle f_{n}, M_{n}^{\prime}\right\rangle\right)-C \sum_{n=1}^{i} \| \mathbb{E}^{\mathcal{A}_{N_{n}}\left[M_{i}^{\prime}\right]-M_{n}^{\prime} \|_{L_{1}}} \\
& \geq \mathbb{E}\left(\sum_{n=1}^{i}\left\langle f_{n}, M_{n}^{\prime}\right\rangle\right)-C \\
& >i \cdot \varepsilon-C .
\end{aligned}
$$

Since $i \in \mathbb{N}$ was arbitrary, $C<\infty$, and $\varepsilon>0$, this contradicts our assumption that $\sup _{i}\left\|M_{i}^{\prime}\right\|_{L_{1}}<\infty$.

Remark 4.4. Proposition 4.3 remains true for unbounded metric spaces $M$. This can be proved using results in the recent preprint [1] where a bounded metric space $\mathcal{B}$ and a linear isomorphism $P: \mathcal{F}(M) \rightarrow \mathcal{F}(\mathcal{B})$ are constructed. It is clear (using [47, Remark on p. 5]) that if $\left(M_{n}\right)_{n=0}^{\infty} \subset L_{1}(\mathcal{F}(M))$ is a bounded martingale adapted to $\left(\mathcal{A}_{n}\right)_{n=0}^{\infty}$ then so is $\left(P M_{n}\right)_{n=0}^{\infty} \subset L_{1}(\mathcal{F}(\mathcal{B}))$, therefore $\left(P M_{n}\right)_{n=0}^{\infty}$ is mean-tight by the bounded versions of Propositions 4.2 and 4.3. Finally, we claim that if $\left(M_{n}\right)_{n=0}^{\infty} \subset L_{1}(\mathcal{F}(\mathcal{B}))$ is mean-tight then $\left(P^{-1} M_{n}\right)_{n=0}^{\infty} \subset L_{1}(\mathcal{F}(M))$ is also mean-tight, which implies the mean Kalton property. This observation requires using the fact that $P^{-1}$ respects supports and compactness (see Proposition 3.2(ix) and Lemma 6.3 in [1]). In fact, we do not know whether there is a direct argument for this claim with the mean Kalton property in place of mean tightness.

We may now deduce the compact determination of the RNP as a consequence of the two previous results. 
Corollary 4.5. The Lipschitz-free space $\mathcal{F}(M)$ has the Radon-Nikodým property if and only if $\mathcal{F}(K)$ has the Radon-Nikodým property for every compact $K \subset M$.

Proof. Since the RNP passes to subspaces, the "only if" implication is immediate. We will prove the contrapositive of the "if" direction.

Let us first remark that we only need to consider the case where $M$ is bounded. Indeed, assume $\mathcal{F}(M)$ fails to have the RNP. Thanks to [38, Proposition 4.3], $\mathcal{F}(M)$ is isomorphic to a subspace of $\left(\sum_{k \in \mathbb{Z}} \mathcal{F}\left(M_{k}\right)\right)_{\ell_{1}}$ for certain bounded subsets $M_{k}$ of $M$. Since the RNP is stable through $\ell_{1}$-sums, isomorphisms, and passing to subspaces, $\mathcal{F}\left(M_{k}\right)$ must fail the RNP for some bounded $M_{k} \subset M$.

By [47, Theorem 2.9], there exists a uniformly integrable martingale $\left(M_{n}\right)_{n=0}^{\infty}$ in $L_{1}(\mathcal{F}(M))$ that does not converge in $L_{1}(\mathcal{F}(M))$. So there exists some $\delta>0$ such that $\limsup _{n, m \rightarrow \infty}\left\|M_{n}-M_{m}\right\|_{L_{1}(\mathcal{F}(M))}>\delta$. The martingale $\left(M_{n}\right)_{n}$ has the mean

Kalton property by Proposition 4.3, so we may apply Proposition 4.2 with $\varepsilon=\frac{\delta}{4}$ to get a compact $K \subset M$, operators $T_{k}: \mathcal{F}(M) \rightarrow \mathcal{F}(M)$ and a map $T:\left(M_{n}\right)_{n} \rightarrow$ $L_{1}(\mathcal{F}(K))$ such that

$$
\sup _{n \in \mathbb{N}}\left\|M_{n}-T\left(M_{n}\right)\right\|_{L_{1}(\mathcal{F}(M))} \leq \frac{\delta}{4}
$$

and

$$
\lim _{k \rightarrow \infty} \sup _{n \in \mathbb{N}}\left\|T_{k}\left(M_{n}\right)-T\left(M_{n}\right)\right\|_{L_{1}(\mathcal{F}(M))}=0
$$

Let us see that $\left(T\left(M_{n}\right)\right)_{n=0}^{\infty}$ is a martingale. Let $\left(\mathcal{A}_{n}\right)$ be a filtration with respect to which $\left(M_{n}\right)$ is a martingale. Since $T_{k}$ commutes with $\mathbb{E}^{\mathcal{A}_{n}}$ for every $k \geq 1$ and $n \geq 0$, and since $T_{k}\left(M_{n}\right) \rightarrow T\left(M_{n}\right)$ for every $n \geq 0$, it follows that

$$
\mathbb{E}^{\mathcal{A}_{n}}\left[T\left(M_{n+1}\right)\right]=\lim _{k \rightarrow \infty} T_{k}\left(\mathbb{E}^{\mathcal{A}_{n}}\left[M_{n+1}\right]\right)=\lim _{k \rightarrow \infty} T_{k}\left(M_{n}\right)=T\left(M_{n}\right) .
$$

Now notice that $\left(T\left(M_{n}\right)\right)$ is $L_{1}(\mathcal{F}(K))$-bounded by $(6)$, since $\left(M_{n}\right)$ is $L_{1}(\mathcal{F}(M))$ bounded. Moreover, the sequence $\left(T_{k}\left(M_{n}\right)\right)_{n=0}^{\infty}$ is uniformly integrable for each $k$ since $T_{k}$ is bounded. Condition $(7)$ then implies that $\left(T\left(M_{n}\right)\right)$ is an $L_{1}(\mathcal{F}(K))$ uniformly integrable martingale. But we have

$$
\limsup _{n, m \rightarrow \infty}\left\|T\left(M_{n}\right)-T\left(M_{m}\right)\right\|_{L_{1}(\mathcal{F}(K))}>\frac{\delta}{2}
$$

by (6), so it cannot converge in $L_{1}(\mathcal{F}(K))$. According to [47, Theorem 2.9] again, this shows that $\mathcal{F}(K)$ fails to have the RNP, completing the proof.

4.2. The structure of Lipschitz-free spaces over purely 1-unrectifiable metric spaces. In general Banach spaces we have

$$
\text { Schur property } \Longrightarrow \text { non-containment of } L_{1} \Longleftarrow \mathrm{RNP}
$$

but all implications absent in the diagram fail in general (either trivially or by deep examples due to Hagler [29] and Bourgain and Rosenthal [10] of spaces with the Schur property failing the RNP). The situation is quite different for Lipschitz-free spaces, as the three properties above are in fact equivalent.

Theorem 4.6. Let $M$ be a metric space. Then the following are equivalent:

(i) The completion of $M$ is purely 1-unrectifiable,

(ii) $\mathcal{F}(M)$ has the Radon-Nikodým property,

(iii) $\mathcal{F}(M)$ has the Krein-Milman property,

(iv) $\mathcal{F}(M)$ has the Schur property,

(v) $\mathcal{F}(M)$ contains no isomorphic copy of $L_{1}$.

(vi) There exists $\varepsilon>0$ such that $\mathcal{F}(M)$ contains no $(1+\varepsilon)$-isomorphic copy of $L_{1}$. 
Proof. By the basic properties of free spaces, the free space over $M$ and the free space over the completion of $M$ are the same, so it suffices to assume $M$ is complete. Property (i) is compactly determined by basic measure theory, and (ii) and (iv) are compactly determined by virtue of Corollary 4.5 and [2, Corollary 2.6], respectively. Thus they are equivalent by Theorem 3.1.

Implication $(\mathrm{v}) \Rightarrow(\mathrm{vi})$ is trivial, and we'll prove (vi) $\Rightarrow$ (i) by contrapositive. Suppose $M$ is not purely 1-unrectifiable, and let $\gamma: K \rightarrow M$ be a curve fragment. By [39, Lemma 4], for every $\varepsilon>0$, there exists a positive measure subset $A \subset K$ such that $\gamma \uparrow_{A}: A \rightarrow M$ is a $(1+\varepsilon)$-bi-Lipschitz embedding. Whenever $A \subset \mathbb{R}$ has positive measure, Godard's theorem [27, proof of Corollary 3.4] implies $\mathcal{F}(A)$ contains an isometric copy of $L_{1}$. Since a $(1+\varepsilon)$-bi-Lipschitz embedding $A \hookrightarrow M$ extends to a $(1+\varepsilon)$-linear isomorphic embedding $\mathcal{F}(A) \hookrightarrow \mathcal{F}(M)$, the conclusion follows.

Finally, implications (ii) $\Rightarrow($ iii $) \Rightarrow(v)$ are true in general Banach spaces, as the Krein-Milman property is hereditary and preserved by isomorphisms, and it fails to hold in $L_{1}$.

Remark 4.7. The argument for equivalence of (iii) can be generalized. Indeed, the conditions in the previous theorem are also equivalent to any other property of $\mathcal{F}(M)$ that lies between non-containment of $L_{1}$ and either the RNP or the Schur property, such as the following:

- The point of continuity property (PCP). Recall that a Banach space $X$ has the PCP provided every non-empty weakly closed and bounded subset admits a point of continuity of the identity map from the weak to the norm topology (see e.g. [23, Section 4]).

- The uniform Kadec-Klee property (UKK). Recall that $X$ is UKK if for each $\varepsilon>0$ there exists $\delta>0$ such that every $\varepsilon$-separated weakly convergent sequence in the closed unit ball of $X$ converges to an element of norm less than $1-\delta[34]$.

\section{A Rectifiable-Connectedness Based Characterization of 1-Critical Sets}

The first main result of this section is Theorem E, by way of Corollary 5.10. We recall that a compact metric space $M$ is called 1-critical if it supports a nonconstant locally flat Lipschitz function, i.e. if $\operatorname{lip}_{0}(M) \neq\{0\}$.

Theorem E (Corollary 5.10). A compact metric space fails to be 1-critical if and only if it is transfinitely almost-rectifiably-connected.

Let us give an intuitive explanation of the meaning of transfinite almost-rectifiableconnectedness. In [52, Chapter 8], Weaver has defined, for a given metric space $(M, d)$, a pseudometric $d_{\mathcal{L}}$ on $M$ by

$$
d_{\mathcal{L}}(x, y):=\sup \left\{|f(y)-f(x)|: f \in B_{\operatorname{lip}(M)}\right\}
$$

and a metric space $M_{\mathcal{L}}$ obtained by identifying points $x, y \in M$ with $d_{\mathcal{L}}(x, y)=0$. In actuality, Weaver's definitions of $\operatorname{lip}(M)$ and $\left(M_{\mathcal{L}}, d_{\mathcal{L}}\right)$ differ from the ones we give for general metric spaces. However, they agree when $M$ is compact, and we cite results from [52, Chapter 8] only in this case. Clearly, $M$ fails to be 1-critical if and only if $M_{\mathcal{L}}$ is a single point. Thus, describing the distance $d_{\mathcal{L}}$ in terms of the geometry of $(M, d)$ yields the desired geometric characterization of 1-criticality. We achieve this goal in the next subsection, where we define a transfinite sequence of spaces $M_{u r}^{(\alpha)}$ and show that $M_{\mathcal{L}}$ is naturally identified with $M_{u r}^{\left(\omega_{1}\right)}$. The space $M_{u r}^{(0)}$ is simply $M$, and intuitively, each space $M_{u r}^{(\alpha+1)}$ is obtained by collapsing every curve fragment in $M_{u r}^{(\alpha)}$ down to an $\mathcal{H}^{1}$-null set. This process must stabilize 
before the first uncountable ordinal $\omega_{1}$, and hence $M_{u r}^{\left(\omega_{1}\right)}$ has no curve fragments to collapse, i.e., it is purely 1-unrectifiable. Then we use Theorem A to conclude:

Informal Theorem (5.9). $M_{\mathcal{L}}=M_{u r}^{\left(\omega_{1}\right)}$.

See Theorem 5.9 for a precise formulation. This theorem shows that $M$ fails to be 1-critical if and only if $M_{u r}^{\left(\omega_{1}\right)}$ is a single point. We are thus lead to the following definition: $M$ is transfinitely almost-rectifiably-connected if $M_{u r}^{\left(\omega_{1}\right)}$ is a single point. We choose this terminology because the statement " $M_{u r}^{(1)}$ is a single point" is almost equivalent to the statement " $M$ is rectifiably-connected" (but not quite, see Example 5.4).

It is well-known (at least as early as [14]) and easy to check that $\lambda(f(M))=0$ whenever $M$ is $\mathcal{H}^{1}-\sigma$-finite and $f \in \operatorname{lip}(M)$. Hence if $M$ is also connected, then $f$ is constant and thus $M$ fails to be 1-critical. Our second main result in this section is Theorem F (by way of Theorem 5.20), where we obtain a quantitative converse of this statement for bounded turning trees (see Definition 5.18) as a natural application of Theorem 5.9.

Theorem F (Theorem 5.20). Let $(M, d)$ be a 1-bounded turning tree. Then for all $x, y \in M$,

$$
d_{\mathcal{L}}(x, y)=\inf \left\{\mathcal{H}_{\infty}^{1}(A):[x, y] \backslash A \text { is } \mathcal{H}^{1}-\sigma \text {-finite }\right\}
$$

In particular, a bounded turning tree fails to be 1-critical if and only if each of its subarcs is $\mathcal{H}^{1}-\sigma$-finite.

It was actually proved by Choquet in [14] that $\lambda(f(M))=0$ for $\mathcal{H}^{1}$ - $\sigma$-finite $M$ under the weaker hypothesis that $f$ satisfies the pointwise flatness condition

$$
\lim _{y \rightarrow x} \frac{|f(y)-f(x)|}{d(x, y)}=0
$$

for every $x \in M$. Thus, Theorem $\mathrm{F}$ shows that a bounded turning tree is 1critical if and only if it supports a nonconstant pointwise flat function, reproving [16, Theorem 2.2]. Theorem F also generalizes a result of Norton [43, Theorem 3] who proved that quasiarcs of Hausdorff dimension strictly larger than one are 1critical.

In the final subsection, we provide examples of spaces $M$ for which the transfinite sequence $M=M_{u r}^{(0)} \rightarrow M_{u r}^{(1)} \rightarrow M_{u r}^{(2)} \rightarrow \ldots$ stabilizes after one step and examples for which the sequence does not stabilize after one step. We also introduce curve-flat Lipschitz functions and use them as a tool for proving non-stabilization.

5.1. $M_{\mathcal{L}}=M_{u r}^{\left(\omega_{1}\right)}$.

Definition 5.1. Let $(M, d)$ be a metric space. We define a pseudometric $d_{u r}$ on $M$ by

$$
d_{u r}(x, y):=\inf _{K} \lambda([\min (K), \max (K)] \backslash K)
$$

where the infimum is over all compact $K \subset \mathbb{R}$ such that there exists a 1-Lipschitz map $\gamma: K \rightarrow M$ with $\gamma(\min (K))=x$ and $\gamma(\max (K))=y$. After identifying any points $x, y$ with $d_{u r}(x, y)=0$, we obtain a metric space $\left(M_{u r}, d_{u r}\right)$ and a canonical 1-Lipschitz surjection $q: M \rightarrow M_{u r}$. The surjection is 1-Lipschitz because for any $x, y \in M$, we may take $K=\{0, d(x, y)\}, \gamma(0)=x$, and $\gamma(d(x, y))=y$, and thus $d(x, y)$ belongs to the set whose infimum equals $d_{u r}(x, y)$. When we wish to emphasize the domain $M$ of the map $q$, we will write $q^{M}$. Whenever $N$ is a second metric space and $f: M \rightarrow N$ is a 1-Lipschitz map, there is a canonically induced 1-Lipschitz map $f_{u r}: M_{u r} \rightarrow N_{u r}$ defined by $f_{u r}\left(q^{M}(x)\right):=q^{N}(f(x))$. It is easy to verify that this is a well-defined 1-Lipschitz map and that the functorial property $(f \circ g)_{u r}=f_{u r} \circ g_{u r}$ holds. 
Remark 5.2. Let us explain an equivalent characterization of the pseudometric $d_{u r}$ induced by any isometric embedding $M \hookrightarrow X$ into a Banach space. It holds that

$$
d_{u r}(x, y)=\inf _{I, K, \gamma} \lambda\left(I \backslash \gamma^{-1}(K)\right)
$$

where the infimum is over all compact intervals $I \subset \mathbb{R}$, compact subsets $K \subset$ $M$, and 1-Lipschitz curves $\gamma: I \rightarrow X$ with $\gamma(\min (I))=x$ and $\gamma(\max (I))=y$. The equality follows from the fact that for every compact $K \subset \mathbb{R}$ and 1-Lipschitz map $\gamma: K \rightarrow M$, there exists a 1-Lipschitz extension $[\min (K), \max (K)] \rightarrow X$ by interpolating with line segments.

The pseudometric $d_{u r}$ admits yet another equivalent characterization in terms of curve-flat Lipschitz functions - see Definition 5.21 and Proposition 5.22.

As will be proved in Proposition 5.23, the map $q: M \rightarrow M_{u r}$ collapses every curve fragment $\gamma(K)$ down to an $\mathcal{H}^{1}$-null set.

Example 5.3 (Subsets of $\mathbb{R}$ ). It is readily seen that if $M \subset \mathbb{R}$ is compact, then $d_{u r}(x, y)=\left|\int_{x}^{y} 1_{\mathbb{R} \backslash M} d \lambda\right|=|f(x)-f(y)|$, where $f(x):=\int_{0}^{x} 1_{\mathbb{R} \backslash M} d \lambda$. Since $\lambda(f(M))=0, M_{u r}$ is isometric to a $\lambda$-null subset of $\mathbb{R}$ and hence is purely 1 unrectifiable.

Example 5.4 (Topologist's Sine Curve). Obviously, if $(M, d)$ is rectifiably-connected, $d_{u r} \equiv 0$ and $M_{u r}$ is a single point. The converse statement is false, and the topologist's sine curve

$$
(\{0\} \times[0,1]) \cup\left\{\left(x, \sin \left(\frac{1}{x}\right)\right) \in \mathbb{R}^{2}: x \in(0,1]\right\} \subset \mathbb{R}^{2}
$$

is a counterexample. For any $y \in\left\{\left(x, \sin \left(\frac{1}{x}\right)\right) \in \mathbb{R}^{2}: x \in(0,1]\right\}, z \in\{0\} \times[0,1]$, and $\varepsilon>0$, there is a rectifiable curve starting at $y$ and ending within a distance $\varepsilon$ from $z$. However, the length of this curve necessarily goes to $\infty$ as $\varepsilon \rightarrow 0$, and this prevents true rectifiable-connectedness.

Proposition 5.5. Let $(M, d)$ be a metric space. Then $q: M \rightarrow M_{u r}$ is an isometry if and only if $M$ is purely 1-unrectifiable.

Proof. Assume $q: M \rightarrow M_{u r}$ is not an isometry. Then there exist $x, y \in M$ such that $d_{u r}(x, y)<d(x, y)$. Isometrically embed $M$ into a Banach space $X$. Then there exist a compact interval $I \subset \mathbb{R}$, a compact subset $K \subset M$, and a 1-Lipschitz curve $\gamma: I \rightarrow X$ such that $\gamma(\min (I))=x, \gamma(\max (I))=y$, and $\lambda\left(I \backslash \gamma^{-1}(K)\right)<d(x, y)$. Then we have

$$
\begin{aligned}
d(x, y) \leq \mathcal{H}^{1}(\gamma(I)) & =\mathcal{H}^{1}(\gamma(I) \cap K)+\mathcal{H}^{1}(\gamma(I) \backslash K) \\
& \leq \mathcal{H}^{1}(\gamma(I) \cap K)+\lambda\left(I \backslash \gamma^{-1}(K)\right) \\
& <\mathcal{H}^{1}(\gamma(I) \cap K)+d(x, y)
\end{aligned}
$$

where the second-to-last inequality follows from the fact that $\gamma$ is 1-Lipschitz. This shows $\mathcal{H}^{1}(\gamma(I) \cap K)>0$ and hence $M$ is not purely 1-unrectifiable.

Now assume $M$ is not purely 1-unrectifiable. Then there exists a compact $K^{\prime} \subset \mathbb{R}$ with $\lambda\left(K^{\prime}\right)>0$ and a bi-Lipschitz embedding $\gamma: K^{\prime} \rightarrow M$. By precomposing with a dilation, we may assume $\gamma$ is 1 -Lipschitz and $\gamma^{-1}$ is $L$-Lipschitz for some $L \in[1, \infty)$. By Lebesgue's density theorem, there exist $t \in K^{\prime}$ and $r>0$ such that $\lambda\left([t, t+r] \backslash K^{\prime}\right)<\frac{r}{4 L}$. Set $K:=[t, t+r] \cap K^{\prime}$. It must hold that $\left[t, t+\frac{r}{4 L}\right] \cap K^{\prime} \neq \emptyset$ and $\left[t+r-\frac{r}{4 L}, t+r\right] \cap K^{\prime} \neq \emptyset$, because otherwise we would have $\lambda\left([t, t+r] \backslash K^{\prime}\right) \geq \frac{r}{2 L}$. This implies $\max (K)-\min (K) \geq r-\frac{r}{2 L} \geq \frac{r}{2}$, and of course we have a fortiori that $\lambda([\min (K), \max (K)] \backslash K)<\frac{r}{4 L}$. Then set $x:=\gamma(\min (K))$ and $y:=\gamma(\max (K))$. We have a compact $K \subset \mathbb{R}$ and a 1-Lipschitz map $\gamma: K \rightarrow M$ such that $\gamma(\min (K))=x$, $\gamma(\max (K))=y$, and $\lambda([\min (K), \max (K)] \backslash K)<\frac{r}{4 L}$, showing $d_{u r}(x, y) \leq \frac{r}{4 L}$. But also, the facts that $\max (K)-\min (K) \geq \frac{r}{2}$ and $\gamma^{-1}$ is $L$-Lipschitz imply $d(x, y) \geq \frac{r}{2 L}$. Hence, $d_{u r}(x, y)<\frac{r}{4 L}<\frac{r}{2 L} \leq d(x, y)$, and $q$ is not an isometry. 
Definition 5.6. Let $(M, d)$ be a metric space. We recursively define a transfinite sequence of metric spaces $\left(M_{u r}^{(\alpha)}, d_{u r}^{(\alpha)}\right)$ and 1-Lipschitz surjections $q_{\alpha}: M \rightarrow M_{u r}^{(\alpha)}$. First, define $\left(M_{u r}^{(0)}, d_{u r}^{(0)}\right)$ to be $(M, d)$ and $q_{0}$ to be the identity map on $M$. Next, fix an ordinal $\alpha>0$ and suppose the definition has been made for all $\alpha^{\prime}<\alpha$. If $\alpha$ is a successor, we define $\left(M_{u r}^{(\alpha)}, d_{u r}^{(\alpha)}\right):=\left(\left(M_{u r}^{(\alpha-1)}\right)_{u r},\left(d_{u r}^{(\alpha-1)}\right)_{u r}\right)$ and $q_{\alpha}:=q \circ q_{\alpha-1}$ where $q=q^{M_{u r}^{(\alpha-1)}}$ is the 1-Lipschitz surjection $M_{u r}^{(\alpha-1)} \rightarrow\left(M_{u r}^{(\alpha-1)}\right)_{u r}$. If $\alpha$ is a limit ordinal, we define a pseudometric $d_{u r}^{(\alpha)}$ on $M$ by

$$
d_{u r}^{(\alpha)}(x, y):=\inf _{\alpha^{\prime}<\alpha} d_{u r}^{\left(\alpha^{\prime}\right)}\left(q_{\alpha^{\prime}}(x), q_{\alpha^{\prime}}(y)\right)
$$

After identifying any points $x, y$ with $d_{u r}^{(\alpha)}(x, y)=0$, we obtain a metric space $\left(M_{u r}^{(\alpha)}, d_{u r}^{(\alpha)}\right)$ and a canonical 1-Lipschitz surjection $q_{\alpha}: M \rightarrow M_{u r}^{(\alpha)}$. When we wish to emphasize the domain $M$, we write $q_{\alpha}^{M}$. Whenever $N$ is a second metric space and $f: M \rightarrow N$ is 1-Lipschitz, we get induced 1-Lipschitz maps $f_{u r}^{(\alpha)}: M_{u r}^{(\alpha)} \rightarrow N_{u r}^{(\alpha)}$ defined by $f_{u r}^{(\alpha)}\left(q_{\alpha}^{M}(x)\right)=q_{\alpha}^{N}(f(x))$. Well-definedness can be verified by transfinite induction, as well as the functorial property $(f \circ g)_{u r}^{(\alpha)}=f_{u r}^{(\alpha)} \circ g_{u r}^{(\alpha)}$.

Let us now see that this iterative process always stabilizes after at most countably many steps when $M$ is separable.

Proposition 5.7. For any separable metric space $M$, there exists a countable ordinal $\alpha_{M}$ such that $q: M_{u r}^{\left(\alpha_{M}\right)} \rightarrow\left(M_{u r}^{\left(\alpha_{M}\right)}\right)_{u r}$ is an isometry.

Proof. Let $(M, d)$ be a separable metric space and $D \subset M$ be a countable dense subset. Fix $(x, y) \in D \times D$. Then we get a nonincreasing map $f: \omega_{1} \rightarrow[0, d(x, y)]$ given by $f(\alpha):=d_{u r}^{(\alpha)}\left(q_{\alpha}(x), q_{\alpha}(y)\right)$. It is easy to see that there is $\alpha_{(x, y)}<\omega_{1}$ such that $f(\alpha)=f\left(\alpha_{(x, y)}\right)$ for all $\alpha \in\left[\alpha_{(x, y)}, \omega_{1}\right)$. Then we set

$$
\alpha_{M}:=\sup \left\{\alpha_{(x, y)}:(x, y) \in D \times D\right\}
$$

and note that $\alpha_{M}$ is countable since $D \times D$ and each $\alpha_{(x, y)}$ are countable. Observe that, for all $(x, y) \in D \times D$,

$$
d_{u r}^{\left(\alpha_{M}+1\right)}\left(q_{\alpha_{M}+1}(x), q_{\alpha_{M}+1}(y)\right)=d_{u r}^{\left(\alpha_{M}\right)}\left(q_{\alpha_{M}}(x), q_{\alpha_{M}}(y)\right)
$$

and thus $q: M_{u r}^{\left(\alpha_{M}\right)} \rightarrow\left(M_{u r}^{\left(\alpha_{M}\right)}\right)_{u r}$ is an isometry restricted to $q_{\alpha_{M}}(D)$. By density and continuity, $q$ must be an isometry on all of $M_{u r}^{\left(\alpha_{M}\right)}$.

When $M$ is 1-rectifiable, $\alpha_{M} \leq 1$. It may happen in general that $\alpha_{M}>1$, and in fact we believe $\alpha_{M}$ can be an arbitrarily large countable ordinal for $M$ compact. See Examples 5.24 and 5.25.

Proposition 5.8. Let $M$ be a separable metric space. Then $M_{u r}^{\left(\omega_{1}\right)}$ is purely 1unrectifiable and satisfies the following universal property: whenever $N$ is a purely 1-unrectifiable metric space and $f: M \rightarrow N$ is a 1-Lipschitz map, there exists a unique 1-Lipschitz map $\tilde{f}: M_{u r}^{\left(\omega_{1}\right)} \rightarrow N$ such that $f=\tilde{f} \circ q_{\omega_{1}}^{M}$.

Proof. That $M_{u r}^{\left(\omega_{1}\right)}$ is purely 1-unrectifiable follows from Propositions 5.5 and 5.7 and transfinite induction. Now let $f: M \rightarrow N$ be a 1-Lipschitz map to a purely 1-unrectifiable metric space $N$. Then we get an induced 1-Lipschitz map $f_{u r}^{\left(\omega_{1}\right)}$ : $M_{u r}^{\left(\omega_{1}\right)} \rightarrow N_{u r}^{\left(\omega_{1}\right)}$. Since $N$ is purely 1-unrectifiable, $q_{\omega_{1}}^{N}: N \rightarrow N_{u r}^{\left(\omega_{1}\right)}$ is an isometry by Proposition 5.5. Then $\tilde{f}:=\left(q_{\omega_{1}}^{N}\right)^{-1} \circ f_{u r}^{\left(\omega_{1}\right)}$ satisfies $f=\tilde{f} \circ q_{\omega_{1}}^{M}$. Uniqueness follows from the surjectivity of $q_{\omega_{1}}^{M}$.

By [52, Corollary 8.13], $\operatorname{lip}\left(M_{\mathcal{L}}\right)$ separates points uniformly when $M$ is bounded, and thus $M_{\mathcal{L}}$ is purely 1 -unrectifiable by Theorem A. Hence, when $M$ is separable, 
the canonical 1-Lipschitz surjection $\pi: M \rightarrow M_{\mathcal{L}}$ induces a 1-Lipschitz map $\tilde{\pi}$ : $M_{u r}^{\left(\omega_{1}\right)} \rightarrow M_{\mathcal{L}}$ by Proposition 5.8. The next theorem is the main one of this section.

Theorem 5.9. For every compact metric space $M$, the map $\tilde{\pi}: M_{u r}^{\left(\omega_{1}\right)} \rightarrow M_{\mathcal{L}}$ is an isometry.

Before proving the theorem, we need to make a small observation. For every metric space $M$, the map $\pi: M \rightarrow M_{\mathcal{L}}$ satisfies the following universal property: whenever $N$ is a metric space for which $\operatorname{lip}(N)$ separates points uniformly with separation constant 1 and $f: M \rightarrow N$ is 1-Lipschitz, there exists a unique 1Lipschitz map $\tilde{f}: M_{\mathcal{L}} \rightarrow N$ such that $f=\tilde{f} \circ \pi$. This observation can be proven directly from the definitions and the fact that $g \circ f \in \operatorname{lip}(M)$ whenever $f: M \rightarrow N$ is Lipschitz and $g \in \operatorname{lip}(N)$.

Proof. Let $M$ be a compact metric space, so that $M_{u r}^{\left(\omega_{1}\right)}$ is also compact because $q_{\omega_{1}}: M \rightarrow M_{u r}^{\left(\omega_{1}\right)}$ is a Lipschitz surjection. Then by Proposition 5.8 and Theorem A, $\operatorname{lip}\left(M_{u r}^{\left(\omega_{1}\right)}\right)$ separates points uniformly. Thus, by the universal property of $\pi: M \rightarrow$ $M_{\mathcal{L}}$, there exists a unique 1-Lipschitz map $\tilde{q}_{\omega_{1}}: M_{\mathcal{L}} \rightarrow M_{u r}^{\left(\omega_{1}\right)}$ such that $\tilde{q}_{\omega_{1}} \circ \pi=$ $q_{\omega_{1}}$.

Since $q_{\omega_{1}}$ is surjective, so is $\tilde{q}_{\omega_{1}}$. Since $\tilde{\pi} \circ q_{\omega_{1}}=\pi$ and $\pi$ is surjective, so is $\tilde{\pi}$. Then we get $\tilde{q}_{\omega_{1}} \circ \tilde{\pi} \circ$ $q_{\omega_{1}}=q_{\omega_{1}}$ and since all these maps are surjective, $\tilde{q}_{\omega_{1}}$ and $\tilde{\pi}$ are inverses. Then since both are 1Lipschitz, both are isometries.

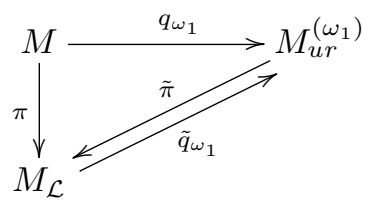

Corollary 5.10. A compact metric space $M$ fails to be 1-critical if and only if $M_{u r}^{\left(\omega_{1}\right)}$ is a single point.

5.2. 1-Critical Bounded Turning Arcs. The object of this subsection is the proof of Theorem F (through Theorem 5.20). We begin by recalling some standard definitions.

A metric space that is homeomorphic to a nonempty, compact interval is called a metric arc. We always assume metric arcs are endowed with an order inherited from a homeomorphism to an interval. Although there are always two such orderings (except if $M$ is a single point), the choice is inconsequential.

A metric arc $(M, d)$ satisfying

$$
d(x, y) \vee d(y, z) \leq d(x, z)
$$

whenever $x \leq y \leq z$ is a 1-bounded turning arc. Equivalently, $\operatorname{diam}([x, z])=d(x, z)$ whenever $x \leq z$. A metric space that is bi-Lipschitz equivalent to a 1-bounded turning arc is called a bounded turning arc. This is equivalent to the existence of a constant $C<\infty$ such that $\operatorname{diam}([x, z]) \leq C d(x, z)$ for every subarc $[x, z]$.

Remark 5.11. Whenever $M$ is a 1-bounded turning arc and $A \subset M$, there exists an interval (equivalently, subarc) $I \supset A$ with $\operatorname{diam}(I)=\operatorname{diam}(A)$. We will use this fact implicitly when dealing with coverings and Hausdorff content of subsets of $M$.

Bounded turning arcs were characterized by Meyer ([41, Corollary 1.2]) as precisely those metric spaces $(M, d)$ for which there exists a homeomorphism $f$ : $[0,1] \rightarrow M$ and $H<\infty$ such that $|x-y| \leq|x-z|$ implies $d(f(x), f(y)) \leq$ $H d(f(x), f(z))$; such a homeomorphism is called a weak quasisymmetry.

The next proposition will be used frequently, and without reference, throughout this subsection. 
Proposition 5.12. For every 1-bounded turning arc $M$, the space $M_{u r}$ is a 1bounded turning arc and $q: M \rightarrow M_{u r}$ is monotone. Even more, for every ordinal $\alpha, M_{u r}^{(\alpha)}$ is a 1-bounded turning arc and $q_{\alpha}: M \rightarrow M_{u r}^{(\alpha)}$ is monotone.

Proof. Let $M$ be a 1-bounded turning arc. For the first part, we begin by showing

$$
d_{u r}(x, y) \vee d_{u r}(y, z) \leq d_{u r}(x, z)
$$

whenever $x \leq y \leq z \in M$. Let $x \leq y \leq z \in M$. Let $\varepsilon>0$, and let $K \subset \mathbb{R}$ be compact and $\gamma: \bar{K} \rightarrow M$ 1-Lipschitz with $\gamma(\min (K))=x, \gamma(\max (K))=z$, and

$$
\lambda([\min (K), \max (K)] \backslash K)<d_{u r}(x, z)+\varepsilon .
$$

If $y \in \gamma(K)$, then $\tilde{K}:=K \cap\left[\min (K), \min \left(\gamma^{-1}(y)\right)\right]$ and $\tilde{\gamma}:=\gamma \uparrow_{\tilde{K}}$ witness $d_{u r}(x, y)<$ $d_{u r}(x, z)+\varepsilon$. If $y \notin \gamma(K)$, then there exist $s, t \in K$ such that

$$
(s, t) \subset[\min (K), \max (K)] \backslash K
$$

and $y \in[\gamma(s), \gamma(t)]$ (or $y \in[\gamma(t), \gamma(s)]$ if $\gamma(t)<\gamma(s)$; assume the former). Then we define $\tilde{K}:=(K \cap[\min (K), s]) \cup\{t\}$ and $\tilde{\gamma}: \tilde{K} \rightarrow M$ by

$$
\tilde{\gamma}(r):= \begin{cases}y & \text { if } r=t \\ \gamma(r) & \text { otherwise. }\end{cases}
$$

Because of the 1-bounded turning property, $\tilde{\gamma}$ is 1-Lipschitz, and thus $\tilde{K}, \tilde{\gamma}$ witness $d_{u r}(x, y)<d_{u r}(x, z)+\varepsilon$. Since $\varepsilon>0$ was arbitrary, we have $d_{u r}(x, y) \leq d_{u r}(x, z)$ in all cases. The other inequality $d_{u r}(y, z) \leq d_{u r}(x, z)$ follows from the same argument. These inequalities imply that the order on $M_{u r}$ defined by $q(x) \leq q(y)$ if $x \leq y$ or $q(x)=q(y)$ is well-defined. Obviously $q$ is monotone and the metric topology on $M_{u r}$ is compact, connected, and agrees with the order topology. It is straightforward to use these facts (for example, with [56, Theorem 28.13]) to prove that $M$ is a 1-bounded turning arc.

The proof of the second part is by transfinite induction. The base case is trivial because $q_{0}$ is the identity map. Let $\alpha>0$ be an ordinal and suppose the proposition holds for all $\alpha^{\prime}<\alpha$. The case where $\alpha$ is a successor follows immediately from the first part, so assume that $\alpha$ is a limit ordinal. Once we show

$$
d_{u r}^{(\alpha)}(x, y) \vee d_{u r}^{(\alpha)}(y, z) \leq d_{u r}^{(\alpha)}(x, z)
$$

whenever $x \leq y \leq z \in M$, the same argument from the first part implies $M_{u r}^{(\alpha)}$ is a 1-bounded turning arc and $q_{\alpha}$ is monotone. Let $x \leq y \leq z \in M$. Let $\varepsilon>0$. Choose $\alpha^{\prime}<\alpha$ large enough so that $d_{u r}^{\left(\alpha^{\prime}\right)}(x, z)<d_{u r}^{(\alpha)}(x, z)+\varepsilon$. By the inductive hypothesis, $d_{u r}^{\left(\alpha^{\prime}\right)}(x, y) \vee d_{u r}^{\left(\alpha^{\prime}\right)}(y, z) \leq d_{u r}^{\left(\alpha^{\prime}\right)}(x, z)$. Together with the inequality from the previous sentence we get $d_{u r}^{(\alpha)}(x, y) \vee d_{u r}^{(\alpha)}(y, z)<d_{u r}^{(\alpha)}(x, z)+\varepsilon$. Since $\varepsilon>0$ was arbitrary, the desired inequality follows.

We shall now work our way towards Theorem $\mathrm{F}$ through a series of lemmas describing the relation between $\mathcal{H}^{1}-\sigma$-finite sets in $M$ and $M_{u r}^{(\alpha)}$ in cases of increasing coverage.

Lemma 5.13. Let $M$ be a 1-bounded turning arc. For every $r>0$ and $\Sigma \subset M_{u r}$, if $\operatorname{diam}(\Sigma)<r$, then there exist subsets $S, A \subset M$ such that $q^{-1}(\Sigma) \subset S \cup A$, $\mathcal{H}_{r}^{1}(A)<r$, and $\mathcal{H}^{1}(S)<\infty$.

Proof. Let $r>0$ and $\Sigma \subset M_{u r}$ with $\operatorname{diam}(\Sigma)<r$. Choose an interval $\left[b_{0}, b_{1}\right] \supset \Sigma$ with $d_{u r}\left(b_{0}, b_{1}\right)=\operatorname{diam}(\Sigma)<r$. By definition of $d_{u r}$, there exist a compact $K \subset \mathbb{R}$ and a 1-Lipschitz map $\gamma: K \rightarrow M$ such that $\gamma(\min (K))=b_{0}, \gamma(\max (K))=b_{1}$, and $\lambda([\min (K), \max (K)] \backslash K)<r$. The set $[\min (K), \max (K)] \backslash K$ is a countable disjoint union of intervals $\left\{\left(x_{i}, y_{i}\right)\right\}_{i}$. The intervals $\left\{\left[\gamma\left(x_{i}\right), \gamma\left(y_{i}\right)\right]\right\}_{i}$ (understood 
to be $\left[\gamma\left(y_{i}\right), \gamma\left(x_{i}\right)\right]$ if $\left.\gamma\left(y_{i}\right) \leq \gamma\left(x_{i}\right)\right)$ together with $\gamma(K)$ cover $\left[b_{0}, b_{1}\right]$. Then $A:=$ $\bigcup_{i}\left[\gamma\left(x_{i}\right), \gamma\left(y_{i}\right)\right]$ and $S:=\gamma(K)$ satisfy the required properties.

Lemma 5.14. Let $M$ be a 1-bounded turning arc. For every $r>0, \delta \in(0, \infty]$, and $\Sigma \subset M_{u r}$, if $\mathcal{H}_{\delta}^{1}(\Sigma)<r$, then there exist subsets $S, A \subset M$ such that $q^{-1}(\Sigma) \subset S \cup A$, $\mathcal{H}_{\delta}^{1}(A)<r$, and $S$ is $\mathcal{H}^{1}-\sigma$-finite.

Proof. Let $r>0, \delta \in(0, \infty]$, and $\Sigma \subset M_{u r}$ with $\mathcal{H}_{\delta}^{1}(\Sigma)<r$. Choose $\varepsilon>0$ such that $\mathcal{H}_{\delta}^{1}(\Sigma)+\varepsilon<r$. Cover $\Sigma$ with countably many sets $\left\{\Sigma_{i}\right\}_{i}$ such that $\operatorname{diam}\left(\Sigma_{i}\right)<\delta$ and $\sum_{i}\left(\operatorname{diam}\left(\Sigma_{i}\right)+2^{-i} \varepsilon\right)<r$. By Lemma 5.13, there exist, for each $i \geq 1$, subsets $S_{i}, A_{i} \subset M$ such that $q^{-1}\left(\Sigma_{i}\right) \subset S_{i} \cup A_{i}, \mathcal{H}_{\delta}^{1}\left(A_{i}\right)<\operatorname{diam}\left(\Sigma_{i}\right)+2^{-i} \varepsilon$, and $\mathcal{H}^{1}\left(S_{i}\right)<\infty$. Then $S:=\bigcup_{i} S_{i}$ and $A:=\bigcup_{i} A_{i}$ satisfy the required properties.

Lemma 5.15. Let $M$ be a 1-bounded turning arc. For every subset $\Sigma \subset M_{\text {ur }}$, if $\Sigma$ is $\mathcal{H}^{1}-\sigma$-finite, then $q^{-1}(\Sigma) \subset M$ is $\mathcal{H}^{1}-\sigma$-finite.

Proof. It suffices to assume $\mathcal{H}^{1}(\Sigma)<\infty$. Let $k \in \mathbb{N}$ be arbitrary. By Lemma 5.14, there exist subsets $S_{k}, A_{k} \subset M$ such that $q^{-1}(\Sigma) \subset S_{k} \cup A_{k}, \mathcal{H}_{1 / k}^{1}\left(A_{k}\right)<\mathcal{H}^{1}(\Sigma)+1$, and $S_{k}$ is $\mathcal{H}^{1}-\sigma$-finite. Set $S:=\bigcup_{k=1}^{\infty} S_{k}$ and $A:=\bigcap_{k=1}^{\infty} A_{k}$. Then $q^{-1}(\Sigma) \subset S \cup A$,

$$
\mathcal{H}^{1}(A)=\sup _{k} \mathcal{H}_{1 / k}^{1}(A) \leq \sup _{k} \mathcal{H}_{1 / k}^{1}\left(A_{k}\right) \leq \mathcal{H}^{1}(\Sigma)+1<\infty,
$$

and $S$ is $\mathcal{H}^{1}-\sigma$-finite. This proves that $q^{-1}(\Sigma)$ is $\mathcal{H}^{1}-\sigma$-finite.

Lemma 5.16. For any ordinal $\alpha$, 1-bounded turning arc $M$ and subsets $\Sigma, B \subset$ $M_{u r}^{(\alpha)}$ such that $\Sigma$ is $\mathcal{H}^{1}-\sigma$-finite, there exist subsets $S, A \subset M$ such that $q_{\alpha}^{-1}(\Sigma \cup$ $B) \subset S \cup A, \mathcal{H}_{\infty}^{1}(A) \leq \mathcal{H}_{\infty}^{1}(B)$ and $S$ is $\mathcal{H}^{1}-\sigma$-finite.

In particular, $q_{\alpha}^{-1}(\Sigma)$ is $\mathcal{H}^{1}-\sigma$-finite whenever $\Sigma$ is $\mathcal{H}^{1}-\sigma$-finite.

Proof. The proof is by transfinite induction. The base case is tautological since $q_{0}$ is the identity map. Let $\alpha$ be an ordinal and suppose the lemma holds for all $\alpha^{\prime}<\alpha$. The case where $\alpha$ is a successor follows immediately from the inductive hypothesis and Lemmas 5.14 and 5.15, so assume $\alpha$ is a limit ordinal. Let $M$ be a 1-bounded turning arc, $r>0$, and $\Sigma, B \subset M_{u r}^{(\alpha)}$ such that $\Sigma$ is $\mathcal{H}^{1}-\sigma$-finite. As in Lemma 5.15, it suffices to assume $\mathcal{H}^{1}(\Sigma)<\infty$. Let $k \in \mathbb{N}$ be arbitrary. Cover $B$ with countably many intervals $\left\{I_{j}^{k}\right\}_{j=1}^{\infty}$ such that $\sum_{j} \operatorname{diam}\left(I_{j}^{k}\right) \leq \mathcal{H}_{\infty}^{1}(B)+2^{-k}$, and similarly cover $\Sigma$ with countably many intervals $\left\{J_{j}^{k}\right\}_{j=1}^{\infty} \operatorname{such}$ that $\operatorname{diam}\left(J_{j}^{k}\right) \leq 2^{-k}$ and $\sum_{j} \operatorname{diam}\left(J_{j}^{k}\right) \leq \mathcal{H}^{1}(\Sigma)+1$. Fix $j \in \mathbb{N}$. Choose $\alpha_{j}<\alpha$ large enough so that

$$
\begin{aligned}
& \operatorname{diam}\left(\left(q_{\alpha}^{\alpha_{j}}\right)^{-1}\left(I_{j}^{k}\right)\right) \leq \operatorname{diam}\left(I_{j}^{k}\right)+2^{-j-k}, \\
& \operatorname{diam}\left(\left(q_{\alpha}^{\alpha_{j}}\right)^{-1}\left(J_{j}^{k}\right)\right) \leq \operatorname{diam}\left(J_{j}^{k}\right)+2^{-j-k},
\end{aligned}
$$

where $q_{\alpha}^{\alpha_{j}}: M_{u r}^{\left(\alpha_{j}\right)} \rightarrow M_{u r}^{(\alpha)}$ denotes the canonical 1-Lipschitz map. This choice is possible because, by the 1-bounded turning condition, the diameter of any interval is determined by only one distance (the distance between the endpoints) and not infinitely many distances.

By the inductive hypothesis,

$$
\begin{aligned}
q_{\alpha}^{-1}\left(I_{j}^{k}\right) & =q_{\alpha_{j}}^{-1}\left(\left(q_{\alpha}^{\alpha_{j}}\right)^{-1}\left(I_{j}^{k}\right)\right) \subset C_{j}^{k} \cup D_{j}^{k} \\
q_{\alpha}^{-1}\left(J_{j}^{k}\right) & =q_{\alpha_{j}}^{-1}\left(\left(q_{\alpha}^{\alpha_{j}}\right)^{-1}\left(J_{j}^{k}\right)\right) \subset E_{j}^{k} \cup F_{j}^{k}
\end{aligned}
$$

where

$$
\begin{aligned}
& \mathcal{H}_{\infty}^{1}\left(C_{j}^{k}\right) \leq \operatorname{diam}\left(I_{j}^{k}\right)+2^{-j-k} \\
& \mathcal{H}_{\infty}^{1}\left(E_{j}^{k}\right) \leq \operatorname{diam}\left(J_{j}^{k}\right)+2^{-j-k} \leq 2^{-k+1}
\end{aligned}
$$

and $D_{j}^{k}, F_{j}^{k}$ are $\mathcal{H}^{1}-\sigma$-finite. Note that this implies $\mathcal{H}_{2^{-k+1}}^{1}\left(E_{j}^{k}\right) \leq \operatorname{diam}\left(J_{j}^{k}\right)+$ $2^{-j-k}$. Set $C^{k}:=\bigcup_{j} C_{j}^{k}, D^{k}:=\bigcup_{j} D_{j}^{k}, E^{k}:=\bigcup_{j} E_{j}^{k}$, and $F^{k}:=\bigcup_{j} F_{j}^{k}$, so that 
- $q_{\alpha}^{-1}(B) \subset C^{k} \cup D^{k}$ and $q_{\alpha}^{-1}(\Sigma) \subset E^{k} \cup F^{k}$,

- $\mathcal{H}_{\infty}^{1}\left(C^{k}\right) \leq \sum_{j}\left(\operatorname{diam}\left(I_{j}^{k}\right)+2^{-j-k}\right) \leq \mathcal{H}_{\infty}^{1}(B)+2^{-k}$

- $\mathcal{H}_{2^{-k+1}}^{1}\left(E^{k}\right) \leq \sum_{j}\left(\operatorname{diam}\left(J_{j}^{k}\right)+2^{-j-k}\right) \leq \mathcal{H}^{1}(\Sigma)+2<\infty$, and

- $D^{k}$ and $F^{k}$ are $\mathcal{H}^{1}-\sigma$-finite.

Set $C:=\bigcap_{k} C^{k}, D:=\bigcup_{k} D^{k}, E:=\bigcap_{k} E^{k}$, and $F:=\bigcup_{k} F^{k}$ so that

- $q_{\alpha}^{-1}(\Sigma \cup B) \subset C \cup D \cup E \cup F$,

- $\mathcal{H}_{\infty}^{1}(C) \leq \inf _{k} \mathcal{H}_{\infty}^{1}\left(C^{k}\right) \leq \mathcal{H}_{\infty}^{1}(B)$,

- $\mathcal{H}^{1}(E)=\sup _{k} \mathcal{H}_{2^{-k+1}}^{1}(E) \leq \sup _{k} \mathcal{H}_{2^{-k+1}}^{1}\left(E^{k}\right) \leq \mathcal{H}^{1}(\Sigma)+2<\infty$, and

- $D$ and $F$ are $\mathcal{H}^{1}-\sigma$-finite.

Then $A:=C$ and $S:=D \cup E \cup F$ satisfy the required properties. This finishes the inductive step and the proof.

The previous lemma is already enough to prove Theorem $\mathrm{F}$ in the particular case of 1-bounded turning arcs.

Theorem 5.17. Let $(M, d)$ be a 1-bounded turning arc with endpoints $x, y$. Then

$$
d_{\mathcal{L}}(\pi(x), \pi(y))=d_{u r}^{\left(\omega_{1}\right)}\left(q_{\omega_{1}}(x), q_{\omega_{1}}(y)\right)=\inf \left\{\mathcal{H}_{\infty}^{1}(A): M \backslash A \text { is } \mathcal{H}^{1}-\sigma \text {-finite }\right\} .
$$

Proof. The first equality follows from Theorem 5.9. Next, observe that applying Lemma 5.16 with $\alpha=\omega_{1}, \Sigma=\emptyset$, and $B=M_{u r}^{\left(\omega_{1}\right)}$ gives us

$$
\begin{aligned}
\inf \left\{\mathcal{H}_{\infty}^{1}(A): M \backslash A \text { is } \mathcal{H}^{1}-\sigma \text {-finite }\right\} \leq \mathcal{H}_{\infty}^{1}\left(M_{u r}^{\left(\omega_{1}\right)}\right) & \leq \operatorname{diam}\left(M_{u r}^{\left(\omega_{1}\right)}\right) \\
& =d_{u r}^{\left(\omega_{1}\right)}\left(q_{\omega_{1}}(x), q_{\omega_{1}}(y)\right) .
\end{aligned}
$$

Finally, we show the reverse inequality. Let $f \in B_{\operatorname{lip}(M)}$ and $A \subset M$ such that $M \backslash A$ is $\mathcal{H}^{1}-\sigma$-finite. Since $M$ is connected, $|f(y)-f(x)| \leq \operatorname{diam}(f(M))=\mathcal{H}_{\infty}^{1}(f(M))$. Then

$$
\begin{aligned}
|f(y)-f(x)| \leq \mathcal{H}_{\infty}^{1}(f(M)) & \leq \mathcal{H}_{\infty}^{1}(f(A))+\mathcal{H}_{\infty}^{1}(f(M \backslash A)) \\
& \leq \mathcal{H}_{\infty}^{1}(A)+\mathcal{H}_{\infty}^{1}(f(M \backslash A))=\mathcal{H}_{\infty}^{1}(A)
\end{aligned}
$$

where the last inequality follows from the fact that $f$ is 1-Lipschitz and the last equality follows from the fact that $f$ is locally flat and $M \backslash A$ is $\mathcal{H}^{1}-\sigma$-finite. Since $A$ and $f$ were arbitrary we get

$$
\begin{aligned}
d_{\mathcal{L}}(\pi(x), \pi(y)) & =\sup \left\{|f(y)-f(x)|: f \in B_{\operatorname{lip}(M)}\right\} \\
& \leq \inf \left\{\mathcal{H}_{\infty}^{1}(A): M \backslash A \text { is } \mathcal{H}^{1} \text { - } \sigma \text {-finite }\right\} .
\end{aligned}
$$

It is now straightforward to extend Theorem 5.17 to the more general class of 1-bounded turning trees, defined below.

Definition 5.18. A compact metric space $M$ is a 1-bounded turning tree if every pair of points $x, y \in M$ are joined by a unique arc in $M$, and this arc is 1-bounded turning. We will denote that arc by $[x, y]$. A metric space is a bounded turning tree if it is bi-Lipschitz equivalent to a 1-bounded turning tree.

Example 5.19. A class of metric spaces called quasiconformal trees was recently studied by Bonk and Meyer in the context of quasisymmetric uniformization. Quasiconformal trees are precisely those bounded turning trees $T$ that are doubling - meaning there exists $N \in \mathbb{N}$ such that for any $r>0$ and $x \in T$, there are $x_{1}, \ldots x_{N} \in T$ with $B_{r}(x) \subset \bigcup_{i=1}^{N} B_{r / 2}\left(x_{i}\right)$. The most well-known examples of quasiconformal trees are Julia sets of polynomials. See [9] for details and more information. 
Theorem 5.20. Let $(M, d)$ be a 1-bounded turning tree. Then for all $x, y \in M$,

$$
d_{\mathcal{L}}(\pi(x), \pi(y))=d_{u r}^{\left(\omega_{1}\right)}\left(q_{\omega_{1}}(x), q_{\omega_{1}}(y)\right)=\inf \left\{\mathcal{H}_{\infty}^{1}(A):[x, y] \backslash A \text { is } \mathcal{H}^{1}-\sigma \text {-finite }\right\} .
$$

In particular, a bounded turning tree fails to be 1-critical if and only if each of its subarcs is $\mathcal{H}^{1}-\sigma$-finite.

Proof. Let $x, y \in M$. The first equality is again Theorem 5.9. The proof of the inequality

$$
d_{\mathcal{L}}(\pi(x), \pi(y)) \leq \inf \left\{\mathcal{H}_{\infty}^{1}(A):[x, y] \backslash A \text { is } \mathcal{H}^{1}-\sigma \text {-finite }\right\}
$$

is just the same as the proof of the analogous inequality from Theorem 5.17. It remains to show the reverse inequality. Fix an order for $[x, y]$ and assume $x \leq$ $y$. For any point $z \in M$, let $g(z)$ denote the first point of contact of $z$ with $[x, y]$. Specifically, $g(z)=\inf \{w \in[z, x]: w \in[x, y]\}$. Then $z \mapsto g(z)$ is a 1 Lipschitz retract onto $[x, y]$ (note that it fixes each element of $[x, y]$ ). This implies the restriction map $B_{\operatorname{lip}(M)} \rightarrow B_{\operatorname{lip}([x, y])}$ is a surjection, because $f \circ g$ maps onto $f$. Together with Theorem 5.17 , this proves

$$
d_{\mathcal{L}}(\pi(x), \pi(y)) \geq \inf \left\{\mathcal{H}_{\infty}^{1}(A):[x, y] \backslash A \text { is } \mathcal{H}^{1}-\sigma \text {-finite }\right\} .
$$

For the second part, observe that the property of being 1-critical and the property of being $\mathcal{H}^{1}-\sigma$-finite is each preserved under bi-Lipschitz maps, and thus it suffices to prove the equivalence when $M$ is a 1-bounded turning tree. If every arc $[x, y] \subset M$ is $\mathcal{H}^{1}-\sigma$-finite, then we may take $A=\emptyset$ in the first part and get $d_{\mathcal{L}}(\pi(x), \pi(y))=0$ for every $x, y \in M$, meaning $M$ is not 1-critical. Conversely, if $M$ is not 1-critical, then $d_{\mathcal{L}}(\pi(x), \pi(y))=0$ for every $x, y \in M$, and so by the first part we may find a sequence $\left\{A_{n}\right\}_{n=1}^{\infty}$ of subsets of $[x, y]$ such that $[x, y] \backslash A_{n}$ is $\mathcal{H}^{1}-\sigma$-finite and $\mathcal{H}_{\infty}^{1}\left(A_{n}\right) \leq \frac{1}{n}$ for every $n \in \mathbb{N}$. This implies $\mathcal{H}_{\infty}^{1}\left(\bigcap_{n=1}^{\infty} A_{n}\right)=0$, and so

$$
[x, y]=\left(\bigcup_{n=1}^{\infty}[x, y] \backslash A_{n}\right) \cup\left(\bigcap_{n=1}^{\infty} A_{n}\right)
$$

is $\mathcal{H}^{1}-\sigma$-finite.

5.3. Curve-Flat Lipschitz Functions. In this final subsection, we define curveflat Lipschitz functions and sketch examples of bounded turning $\operatorname{arcs} M$ whose index $\alpha_{M}$ from Proposition 5.7 may be arbitrarily large.

Definition 5.21 (Curve-Flat Lipschitz Functions). Let $(M, d)$ be a metric space. A Lipschitz function $f: M \rightarrow \mathbb{R}$ is curve-flat if for any compact $K \subset \mathbb{R}$ and Lipschitz $\gamma: K \rightarrow M$, the composite $f \circ \gamma$ is flat at $\lambda$-almost every point in $K$, meaning

$$
\lim _{\substack{y \rightarrow x \\ y \in K}} \frac{|f(\gamma(y))-f(\gamma(x))|}{|x-y|}=0
$$

for $\lambda$-almost every $x \in K$. Equivalently, by the area formula [22, pg. 299], $\lambda(f(\gamma(K)))=0$ for every $K \subset \mathbb{R}$ compact and $\gamma: K \rightarrow M \operatorname{Lipschitz.~Let~} \operatorname{lip}_{\Gamma}(M)$ denote the set of all curve-flat Lipschitz functions on $M$ and $B_{\operatorname{lip}_{\Gamma}(M)}$ the set of curve-flat 1-Lipschitz functions. Define a pseudometric $d_{\Gamma}$ on $M$ by

$$
d_{\Gamma}(x, y):=\sup \left\{|f(y)-f(x)|: f \in B_{\operatorname{lip}_{\Gamma}(M)}\right\} .
$$

Curve-flat Lipschitz functions are intimately connected to the pseudometric $d_{u r}$ by the following proposition.

Proposition 5.22. For any metric space $(M, d)$ and $f \in B_{\operatorname{Lip}\left(M_{u r}\right)}, f \circ q \in$ $B_{\operatorname{lip}_{\Gamma}(M)}$. Consequently, $d_{u r}=d_{\Gamma}$. 


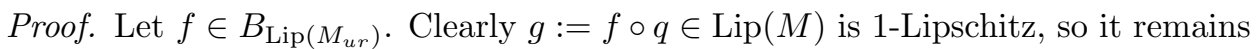
to prove that $g$ is curve-flat. Let $K \subset \mathbb{R}$ be compact and $\gamma: K \rightarrow M$ be 1-Lipschitz, and fix $a \leq b \in K$. Then

$$
\begin{aligned}
|g \circ \gamma(a)-g \circ \gamma(b)| & =|f \circ q \circ \gamma(a)-f \circ q \circ \gamma(b)| \\
& \leq\|f\|_{L} \cdot d_{u r}(q(\gamma(a)), q(\gamma(b))) \\
& \leq d_{u r}(\gamma(a), \gamma(b)) \\
& \leq \lambda([a, b] \backslash K)
\end{aligned}
$$

where we apply the definition of $d_{u r}$ to the restriction of $\gamma$ to $K \cap[a, b]$. By Lebesgue's density theorem, we have $\lambda([a, b] \backslash K) /(b-a) \rightarrow 0$ as $b \rightarrow a$ for $\lambda$-almost every $a \in K$ (or as $a \rightarrow b$ for $\lambda$-almost every $b \in K$ ). Thus $g$ is curve-flat.

The first statement immediately implies $d_{u r} \leq d_{\Gamma}$, and the reverse inequality is a simple consequence of Lebesgue's fundamental theorem of calculus. We omit the details.

We can use Proposition 5.22 to prove that $q$ collapses every curve fragment in $M$ down to an $\mathcal{H}^{1}$-null subset of $M_{u r}$.

Proposition 5.23. For every metric space $M$, compact $K \subset \mathbb{R}$, and Lipschitz $\gamma: K \rightarrow M, \mathcal{H}^{1}(q(\gamma(K)))=0$.

Proof. Suppose the proposition is false. Then by [39, Lemma 4 and Theorem 9], we can find $K \subset \mathbb{R}$ compact with $\lambda(K)>0$ and $\gamma: K \rightarrow M$ Lipschitz such that $q \circ \gamma: K \rightarrow M_{u r}$ is a bi-Lipschitz embedding. Then $(q \circ \gamma)^{-1}: q(\gamma(K)) \rightarrow \mathbb{R}$ is a biLipschitz embedding, and we let $g: M_{u r} \rightarrow \mathbb{R}$ be any McShane-Whitney extension. By Proposition 5.22, $g \circ q: M \rightarrow \mathbb{R}$ is curve-flat Lipschitz, but $\lambda(g(q(\gamma(K))))=$ $\lambda(K)>0$, a contradiction.

We will use the remainder of this subsection to estimate the index $\alpha_{M}$ of some example spaces $M$.

Example 5.24 (1-Rectifiable Metric Spaces). A metric space is 1-rectifiable if it is the union of countably many curve fragments and an $\mathcal{H}^{1}$-null set. Proposition 5.23 implies $\mathcal{H}^{1}\left(M_{u r}\right)=0$ whenever $M$ is 1-rectifiable, hence $M_{u r}$ is purely 1-unrectifiable. In this case, $\alpha_{M}=0$ if $\mathcal{H}^{1}(M)=0$ and $\alpha_{M}=1$ if $\mathcal{H}^{1}(M)>0$.

Every 1-rectifiable metric space is $\mathcal{H}^{1}-\sigma$-finite, and so Example 5.24 may tempt one to believe $\alpha_{M} \leq 1$ whenever $M$ is $\mathcal{H}^{1}-\sigma$-finite. Our final example shows that this is not the case.

Example 5.25 (Bounded Turning Arcs). We will sketch the construction of a bounded turning arc $M$ with $\operatorname{diam}(M)=\operatorname{diam}\left(M_{u r}\right)=1$, but $M_{u r}^{\left(\omega_{1}\right)}$ is a single point, which automatically implies $\alpha_{M}>1$. We believe the construction can be iterated to make $\alpha_{M}$ an arbitrarily large countable ordinal, but we leave those details to the interested reader.

Let $\mathcal{C} \subset[0,1]$ be the standard middle thirds Cantor set and $\beta:=\log _{3}(2)$ the Hausdorff dimension of $\mathcal{C}$. The Cantor function $f: \mathcal{C}^{\beta} \rightarrow[0,1]$ is monotone, surjective, and 1-Lipschitz ([21, Proposition 10.1]), where $\mathcal{C}^{\beta}$ denotes the snowflake space. Let $(M, d)$ be the metric space obtained by "filling in the gaps" of $\mathcal{C}^{\beta}$ with geodesics. Precisely, consider the collection of all doubletons $\{x<y\} \subset \mathcal{C}^{\beta}$ such that $[x, y] \cap \mathcal{C}=\{x, y\}$, then form the disjoint union $\mathcal{C}^{\beta} \sqcup \bigsqcup_{\{x<y\}}[0, d(x, y)]$, identify each $x$ with its copy 0 and each $y$ with $d(x, y)$, and equip the resulting quotient space $M$ with the largest metric $d$ such that the inclusions $\mathcal{C}^{\beta},[0, d(x, y)] \hookrightarrow(M, d)$ are isometric embeddings. The space $M$ is a 1-bounded turning $\operatorname{arc}$ with $\operatorname{diam}(M)=$ 1. The Cantor function $f$ extends to a 1-Lipschitz map $f: M \rightarrow[0,1]$ that is 
constant on each geodesic. Observe that $f$ is curve-flat because $\mathcal{C}^{\beta}$ is purely 1unrectifiable and $f$ is constant on each of the countably many geodesics. This gives us $d_{u r}(x, y)=d_{\Gamma}(x, y) \geq|f(x)-f(y)|$. In particular, $\operatorname{diam}\left(M_{u r}\right)=1$. However, $\mathcal{H}^{1}\left(\mathcal{C}^{\beta}\right)=\mathcal{H}^{\beta}(\mathcal{C})<\infty$, implying that $M$ is $\mathcal{H}^{1}-\sigma$-finite. Thus, $M_{u r}^{\left(\omega_{1}\right)}=M_{\mathcal{L}}$ is a single point by Theorem 5.17 .

\section{ACKNOWLEDGMENTS}

The authors wish to thank Marek Cúth and Gilles Godefroy for some insightful remarks on the topic of the paper.

R. J. Aliaga was partially supported by the Spanish Ministry of Economy, Industry and Competitiveness under Grant MTM2017-83262-C2-2-P. C. Petitjean and A. Procházka were partially supported by the French ANR project No. ANR-20CE40-0006.

\section{REFERENCES}

[1] F. Albiac, J. Ansorena, M. Cúth and M. Doucha, Lipschitz algebras and Lipschitz-free spaces over unbounded metric spaces. Preprint (2020), arXiv:2011.12993.

[2] R. J. Aliaga, C. Noûs, C. Petitjean and A. Procházka, Compact reduction in Lipschitz free spaces, to appear in Studia Math. Preprint (2020), arXiv:2004.14250.

[3] R. J. Aliaga, C. Petitjean and A. Procházka, Embeddings of Lipschitz-free spaces into $\ell_{1}$, J. Funct. Anal. 280 (2021), no. 6, 108916.

[4] R. J. Aliaga, E. Pernecká, C. Petitjean and A. Procházka, Supports in Lipschitz-free spaces and applications to extremal structure, J. Math. Anal. Appl. 489 (2020), 124128.

[5] W. G. Bade, P. C. Curtis, Jr. and H. G. Dales, Amenability and weak amenability for Beurling and Lipschitz algebras, Proc. London Math. Soc. 55 (1987), 359-377.

[6] D. Bate, Structure of measures in Lipschitz differentiability spaces, J. Amer. Math. Soc. 28 (2015), no. 2, 421-482.

[7] D. Bate, Purely unrectifiable metric spaces and perturbations of Lipschitz functions, Acta Math. 224 (2020), 1-65.

[8] D. Bate and S. Li, Characterizations of rectifiable metric measure spaces, Ann. Sci. Éc. Norm. Supér. 50 (2017), no. 1, 1-37.

[9] M. Bonk and D. Meyer, Quasiconformal and geodesic trees, Fund. Math. 250 (2020), no. 3, 253-299.

[10] J. Bourgain, and H. P. Rosenthal, Martingales valued in certain subspaces of $L^{1}$, Israel J. Math. 37, no. 1-2, 54-75.

[11] B. Braga, G. Lancien, C. Petitjean and A. Procházka, On Kalton's interlaced graphs and nonlinear embeddings into dual Banach spaces. Preprint (2019), arXiv:1909.12132.

[12] B. Cascales, R. Chiclana, L. García-Lirola, M. Martín and A. Rueda Zoca, On strongly norm attaining Lipschitz maps, J. Funct. Anal. 277 (2019), no. 6, 1677-1717.

[13] J. Cheeger and B. Kleiner, Differentiability of Lipschitz maps from metric measure spaces to Banach spaces with the Radon-Nikodým property, Geom. Funct. Anal. 19 (2009), no. 4, 1017-1028.

[14] G. Choquet, L'isométrie des ensembles dans ses rapports avec la théorie du contact et la théorie de la mesure, Mathematica (Timişoara) 20 (1944), 29-64.

[15] Ş. Cobzaş, R. Miculescu and A. Nicolae, Lipschitz functions, Lecture Notes in Mathematics, 2241, Springer, Cham, 2019.

[16] M. Csörnyei, J. Kališ, and L. Zajíček, Whitney arcs and 1-critical arcs, Fund. Math. 199 (2008), no. 2, 119-130.

[17] A. Dalet, Free spaces over countable compact metric spaces, Proc. Amer. Math. Soc. 143 (2015), 3537-3546.

[18] A. Dalet, Free spaces over some proper metric spaces, Mediterr. J. Math. 12 (2015), 973-986.

[19] G. David and S. Semmes, Fractured fractals and broken dreams. Self-similar geometry through metric and measure. Oxford Lecture Series in Mathematics and its Applications, 7. The Clarendon Press, Oxford University Press, New York, 1997.

[20] J. Diestel and J. J. Uhl, Jr., Vector measures, Mathematical Surveys, 15. American Mathematical Society, Providence, RI, 1977.

[21] O. Dovgoshey, O. Martio, V. Ryazanov and M. Vuorinen, The Cantor function, Expo. Math. 24 (2006), no. 1, 1-37. 
[22] H. Federer, Colloquium lectures on geometric measure theory, Bull. Amer. Math. Soc. 84 (1978), no. 3, 291-338.

[23] V. P. Fonf, J. Lindenstrauss and R. R. Phelps, Infinite dimensional convexity, Handbook of the geometry of Banach spaces, Vol. I, 599-670. Elsevier, 2001.

[24] L. García-Lirola, C. Petitjean, A. Procházka and A. Rueda Zoca, Extremal structure and duality of Lipschitz free spaces, Mediterr. J. Math. 15 (2018), no. 2, Art. 69.

[25] L. García-Lirola, A. Procházka and A. Rueda Zoca, A characterization of the Daugavet property in spaces of Lipschitz functions, J. Math. Anal. Appl. 464 (2018), no. 1, 473-492.

[26] C. Gartland, Lipschitz free spaces over locally compact metric spaces, to appear in Studia Math. Preprint (2020), arXiv:2004.11951.

[27] A. Godard, Tree metrics and their Lipschitz-free spaces, Proc. Amer. Math. Soc. 138 (2010), 4311-4320.

[28] G. Godefroy, A survey on Lipschitz-free Banach spaces, Comment. Math. 55 (2015), no. 2, 89-118.

[29] J. Hagler, A counterexample to several questions about Banach spaces, Studia Math. 60 (1977), no. 3, 289-308.

[30] P. Hájek, G. Lancien and E. Pernecká, Approximation and Schur properties for Lipschitz free spaces over compact metric spaces, Bull. Belg. Math. Soc. Simon Stevin 23 (2016), 63-72.

[31] P. Hajłasz, Whitney's example by way of Assouad's embedding, Proc. Amer. Math. Soc. 131 (2003), no. 11, 3463-3467.

[32] L. G. Hanin, Kantorovich-Rubinstein norm and its application in the theory of Lipschitz spaces, Proc. Amer. Math. Soc. 115 (1992), 345-352.

[33] P. Harmand, D. Werner, and W. Werner, M-ideals in Banach spaces and Banach algebras, Lecture Notes in Math. 1547, Springer-Verlag, Berlin-Heidelberg, 1993.

[34] R. Huff, Banach spaces which are nearly uniformly convex, Rocky Mountain J. Math. 10 (1980), no. 4, 743-749.

[35] A. Illanes and S. Nadler, Hyperspaces. Fundamentals and recent advances. Monographs and Textbooks in Pure and Applied Mathematics, 216. Marcel Dekker, Inc., New York, 1999.

[36] J. A. Johnson, Banach spaces of Lipschitz functions and vector-valued Lipschitz functions, Trans. Amer. Math. Soc. 148 (1970), 147-169.

[37] V. Kadets and D. Werner, A Banach space with the Schur and the Daugavet property, Proc. Amer. Math. Soc. 132, no. 6, 1765-1773.

[38] N. J. Kalton, Spaces of Lipschitz and Hölder functions and their applications, Collect. Math. 55 (2004), 171-217.

[39] B. Kirchheim, Rectifiable metric spaces: local structure and regularity of the Hausdorff measure, Proc. Amer. Math. Soc. 121 (1994), 113-123.

[40] P. Mattila, Geometry of sets and measures in Euclidean spaces, Cambridge Studies in Advanced Mathematics 44, Cambridge University Press, Cambridge, 1995.

[41] D. Meyer, Bounded turning circles are weak-quasicircles, Proc. Amer. Math. Soc. 139 (2011), no. 5, 1751-1761.

[42] A. Naor and G. Schechtman, Planar earthmover is not in $L_{1}$, SIAM J. Comput. 37 (2007), no. 3, 804-826.

[43] A. Norton, Functions not constant on fractal quasi-arcs of critical points, Proc. Amer. Math. Soc. 106 (1989), no. 2, 397-405.

[44] M. Ostrovskii, Radon-Nikodým property and thick families of geodesics, J. Math. Anal. Appl. 409 (2014), 906-910.

[45] C. Petitjean, Lipschitz-free spaces and Schur properties, J. Math. Anal. Appl. 453 (2017), 894-907.

[46] C. Petitjean, Some aspects of the geometry of Lipschitz-free spaces, PhD Thesis (2018), Univ. Bourgogne Franche-Comté.

[47] G. Pisier, Martingales in Banach spaces, Cambridge University Press, Cambridge, 2016.

[48] A. Procházka and A. Rueda Zoca, A characterization of octahedrality in Lipschitz-free spaces, Ann. Inst. Fourier (Grenoble) 68 (2018), no. 2, 569-588.

[49] C. Villani, Topics in optimal transportation, Graduate Studies in Mathematics, 58, American Mathematical Society, Providence, RI, 2003.

[50] N. Weaver, Subalgebras of little Lipschitz algebras, Pacific J. Math. 173 (1996), 283-293.

[51] N. Weaver, Lipschitz algebras, World Scientific Publishing Co., Inc., River Edge, NJ, 1999.

[52] N. Weaver, Lipschitz algebras, 2nd ed., World Scientific Publishing Co., River Edge, NJ, 2018.

[53] Z.-Y. Wen and L.-F. Xi, The geometry of Whitney's critical sets, Israel J. Math. 174 (2009), 303-348.

[54] H. Whitney, Analytic extensions of differentiable functions defined in closed sets, Trans. Amer. Math. Soc. 36 (1934), 63-89. 
[55] H. Whitney, A function not constant on a connected set of critical points, Duke Math. J. 1 (1935), no. 4, 514-517.

[56] S. Willard, General Topology, Addison-Wesley Publishing Co., Reading, Mass.-London-Don Mills, Ont., 1970.

(R. J. Aliaga) Instituto Universitario de Matemática Pura y Aplicada, Universitat Politècnica de València, Camino de Vera S/N, 46022 Valencia, Spain

Email address: raalva@upvnet.upv.es

(C. Gartland) Texas A\&M University, College Station, TX 77843, USA

Email address: cgartland@math.tamu.edu

(C. Petitjean) LAma, Univ Gustave Eiffel, UPem, Univ Paris Est Creteil, CNRS, F-77447, Marne-La-VAllée, France

Email address: colin.petitjean@univ-eiffel.fr

(A. Procházka) Laboratoire de Mathématiques de Besançon, Université Bourgogne Franche-Comté, CNRS UMR-6623, 16, route de Gray, 25030 Besançon Cedex, France Email address: antonin.prochazka@univ-fcomte.fr 